\title{
Nanoscale Mapping of Non-Uniform Heterogeneous Nucleation Kinetics Mediated by Surface Chemistry
}

\author{
Mei Wang, Thilini Umesha Dissanayake, Chiwoo Park, Karen J. Gaskell, Taylor Woehl
}

Submitted date: 25/07/2019 - Posted date: 26/07/2019

Licence: CC BY-NC-ND 4.0

Citation information: Wang, Mei; Dissanayake, Thilini Umesha; Park, Chiwoo; Gaskell, Karen J.; Woehl, Taylor (2019): Nanoscale Mapping of Non-Uniform Heterogeneous Nucleation Kinetics Mediated by Surface Chemistry. ChemRxiv. Preprint.

Nucleation underlies the formation of many liquid-phase synthetic and natural materials with applications in materials chemistry, geochemistry, biophysics, and structural biology. Most liquid-phase nucleation processes are heterogeneous, occurring at specific nucleation sites at a solid-liquid interface; however, the chemical and topographical identity of these nucleation sites and how nucleation kinetics vary from site-to-site remains mysterious. Here we utilize in situ liquid cell electron microscopy to unveil counterintuitive nanoscale non-uniformities in heterogeneous nucleation kinetics on a macroscopically uniform solid-liquid interface. Time-resolved in situ electron microscopy imaging of silver nanoparticle nucleation at a water-silicon nitride interface showed apparently randomly-located nucleation events at the interface. However, nanometric maps of local nucleation kinetics uncovered nanoscale interfacial domains with either slow or rapid nucleation. Interestingly, the interfacial domains vanished at high supersaturation ratio, giving way to rapid spatially uniform nucleation kinetics. Atomic force microscopy and nanoparticle labeling experiments revealed a topographically flat, chemically heterogeneous interface with nanoscale interfacial domains of functional groups similar in size to those observed in the nanometric nucleation maps. These results, along with a semi-quantitative nucleation model, indicate that a chemically non-uniform interface presenting different free energy barriers to heterogeneous nucleation underlies our observations of non-uniform nucleation kinetics. Overall, our results introduce a new imaging modality, nanometric nucleation mapping, and provide important new insights into the impact of surface chemistry on microscopic spatial variations in heterogeneous nucleation kinetics that have not been previously observed.

File list (2)

Manuscript_1strevision_submit_forChemRxiv.pdf (1.73 MiB) view on ChemRxiv - download file 
Nanoscale mapping of non-uniform heterogeneous nucleation kinetics mediated by surface chemistry

Mei Wang, ${ }^{1}$ Thilini U. Dissanayake, ${ }^{1}$ Chiwoo Park, ${ }^{2}$ Karen Gaskell, ${ }^{3}$ Taylor J. Woehl ${ }^{1, *}$

${ }^{1}$ Department of Chemical and Biomolecular Engineering, University of Maryland, College Park, Maryland 20742, United States

${ }^{2}$ Department of Industrial and Manufacturing Engineering, Florida State University, Tallahassee, Florida 32306, United States

${ }^{3}$ Department of Chemistry \& Biochemistry, University of Maryland, College Park, Maryland 20742, United States

*Corresponding author, email: tjwoehl@umd.edu

\section{Abstract}

Nucleation underlies the formation of many liquid-phase synthetic and natural materials with applications in materials chemistry, geochemistry, biophysics, and structural biology. Most liquidphase nucleation processes are heterogeneous, occurring at specific nucleation sites at a solidliquid interface; however, the chemical and topographical identity of these nucleation sites and how nucleation kinetics vary from site-to-site remains mysterious. Here we utilize in situ liquid cell electron microscopy to unveil counterintuitive nanoscale non-uniformities in heterogeneous nucleation kinetics on a macroscopically uniform solid-liquid interface. Time-resolved in situ electron microscopy imaging of silver nanoparticle nucleation at a water-silicon nitride interface showed apparently randomly-located nucleation events at the interface. However, nanometric maps of local nucleation kinetics uncovered nanoscale interfacial domains with either slow or rapid nucleation. Interestingly, the interfacial domains vanished at high supersaturation ratio, giving way to rapid spatially uniform nucleation kinetics. Atomic force microscopy and nanoparticle labeling experiments revealed a topographically flat, chemically heterogeneous interface with nanoscale interfacial domains of functional groups similar in size to those observed in the nanometric nucleation maps. These results, along with a semi-quantitative nucleation model, indicate that a 
chemically non-uniform interface presenting different free energy barriers to heterogeneous nucleation underlies our observations of non-uniform nucleation kinetics. Overall, our results introduce a new imaging modality, nanometric nucleation mapping, and provide important new insights into the impact of surface chemistry on microscopic spatial variations in heterogeneous nucleation kinetics that have not been previously observed.

\section{Introduction}

Nucleation is the first step in a liquid to solid phase transition and as such is an important and ubiquitous process in natural and synthetic chemical and physical processes. Nucleation mediates diverse processes, such as ice and cloud formation in the atmosphere, ${ }^{1}$ polymorph control during crystallization of pharmaceuticals, ${ }^{2}$ chemical synthesis of nanocrystals, ${ }^{3}$ and biomineralization. ${ }^{4,5}$ Despite its universal occurrence and critical role in influencing properties of a crystallizing solid phase, nucleation remains poorly understood.

Most nucleation processes are heterogeneous, occurring at an interface that lowers the free energy barrier for nucleation. ${ }^{6}$ The topology and chemistry of the interface both mediate the heterogeneous nucleation kinetics. For example, porous materials have been used to concentrate protein molecules to form nuclei, where the pore geometry and size greatly influence nucleation rate. ${ }^{6-8}$ Surface chemistry has also been demonstrated to impact heterogenous nucleation kinetics, where functional groups can serve as nucleation sites. ${ }^{6,9,10}$ For instance, binding of precursor ions to certain functional groups has been shown to increase nucleation rate of calcium carbonate by increasing the local supersaturation ratio ${ }^{10}$ or decreasing the overall interfacial energy of the nuclei. ${ }^{11}$ However, it remains challenging to identify precisely how the nature of heterogeneous nucleation sites impacts nucleation kinetics, due to the lack of experimental characterization methods capable of identifying discrete nucleation events at the salient time and spatial scales. 
Nucleation is difficult to probe because nuclei are sub-nanometer in size and appear stochastically and transiently in time.${ }^{12}$ Current experimental approaches are limited in visualizing where and when nucleation occurs. Nucleation kinetics can be inferred from optical microscopy, ${ }^{9}$ but one must assume constant growth rate over the time it takes the nuclei to reach observable size. In situ atomic force microscopy (AFM) has long been utilized to observe nucleation at solid-liquid interfaces, primarily for protein and molecular crystallization. ${ }^{13-18}$ However, the resolution of AFM is still low $(\sim 10 \mathrm{~nm})$ compared to the expected sub-nanometer size of inorganic nuclei and has poor time resolution except in all but the newest generation of fast AFMs, limiting its ability to isolate nucleation in time and space. Liquid cell transmission electron microscopy (LC-TEM) is an emerging and promising approach for visualizing nucleation due to its high temporal and spatial resolution. Most research utilizing LC-TEM has focused on growth ${ }^{19-22}$ or etching dynamics ${ }^{23-25}$ of nanoparticles with only a few studies using LC-TEM to observe nucleation. ${ }^{26-28}$ Further, understanding of the electron beam induced changes to solution chemistry during LC-TEM imaging is still in nascent stages, ${ }^{27,29-33}$ making control over important nucleation parameters like supersaturation ratio difficult.

In this article, we utilize liquid cell scanning transmission electron microscopy (LC-STEM) to spatially map heterogeneous nucleation kinetics of silver nanocrystals at a solid/liquid interface. In a previous article, ${ }^{27}$ we established a controllable and reproducible solution chemistry for LCSTEM and determined that ensemble nucleation rate of silver nanocrystals was proportional only to LC-STEM imaging magnification, opening the door to utilize this imaging parameter as a surrogate for supersaturation ratio. Here, by correlating the nucleation kinetics of individual nanoparticles with their spatial position at the interface, we have discovered silver nanoparticle nucleation on a macroscopically uniform silicon nitride-water interface proceeds via preferential 
heterogeneous nucleation on nanoscale interfacial domains of functional groups. Chemical force microscopy (CFM) and nanoparticle labeling experiments revealed hydrophilic domains of functional groups on the interface with similar size as the domains observed in the nucleation maps. This mechanism is further supported by a phenomenological nucleation model based on classical nucleation theory (CNT). More broadly, our results directly show that microscopic variations in surface chemistry on a macroscopically homogeneous surface can lead to microscopic variations in heterogeneous nucleation kinetics. ${ }^{9,34,35}$ This new insight has broad implications for processes where heterogeneous nucleation is important, including biomineralization, 4, 5, 36, 37 nanomaterial synthesis (e.g. seeded nanoparticle synthesis, supported catalyst synthesis), ${ }^{38-41}$ and hybrid inorganic-organic material synthesis (e.g. MOFS, halide perovskites). ${ }^{42-50}$

\section{Results and Discussion}

The nucleation of silver nanocrystals was visualized in real-time by low magnification LCSTEM imaging (Figure 1a-d). Here the electron beam reduces silver ions into silver atoms, which become supersaturated in solution causing nucleation and growth. ${ }^{29}$ To avoid oxidative etching of nuclei, experiments were carried out in a strong reducing environment created by aqueous electrons and hydrogen radicals, with $0.1 \mathrm{M}$ tert-butanol added to scavenge oxidizing species. ${ }^{27}$ Quantitative analysis of the LC-STEM movies showed the number of silver nanocrystals increased over several minutes with an overall rate that increased with the image magnification (Figure 1e). We note that we do not directly visualize nuclei but instead detect them indirectly once they reach a size of $\sim 4 \mathrm{~nm}$ in diameter. Nanocrystals were immobile throughout the nucleation and growth process, suggesting they formed by heterogeneous nucleation at the solid-liquid interface between the silicon nitride membrane and liquid. A single nucleation rate was not observed for each 
condition (cf. Figure 1e), so instead we quantified the nucleation induction time $\left(t_{\text {ind }}\right)$ of each nanocrystal, which is defined as the time between the start of LC-STEM imaging and the time required for a particle to grow to a detectible size. ${ }^{28}$ Nucleation induction time is inversely proportional to nucleation rate and is a common metric for quantifying nucleation kinetics when there is insufficient spatial/temporal resolution to directly identify nuclei. ${ }^{12}$ Figure $1 \mathbf{f}$ shows the nucleation induction time distribution for three different LC-STEM magnifications. The distribution shifts to shorter induction times with increasing magnification, indicating more rapid formation of nuclei. The median nucleation induction time decreased nearly linearly with increasing LC-STEM magnification and was inversely proportional to the initial silver ion concentration (Figure 1g). Taken together, these results indicate the nucleation rate was proportional to both image magnification and precursor concentration, suggesting these two parameters are directly proportional to the supersaturation ratio. While we cannot directly determine the supersaturation ratio of silver atoms in these experiments, it is approximated to be on the order of $S=\frac{C_{A g}}{C_{A g, 0}}=10^{7}$ (see Supporting information methods for derivation). This value is similar to a previous LC-TEM study on multi-step nanoparticle nucleation ${ }^{26}$ and estimates for flask-based nanocrystal synthesis. ${ }^{51}$

For a given supersaturation ratio, some nanocrystals clearly nucleate more rapidly than others, but a cursory look at the time lapsed LC-STEM images in Figure 1a-d would make it seem that nucleation occurs at random spatial locations on the solid/liquid interface. This idea is further supported by the seemingly flat, homogeneous interface created between the microfabricated silicon nitride and liquid. However, a more detailed look revealed spatially varying nucleation kinetics across the interface. Figure 2 shows nucleation flux maps, where the black dots represent nanocrystal centroids measured from LC-STEM movies and the heat map is an interpolated surface 
showing the local nucleation flux (nuclei/area/time) (see Supporting information for methods of generating nucleation flux maps). For relatively low supersaturation ratio (Figure 2a), few nuclei were observed initially, giving a low nucleation flux across the interface. As the reaction progressed, nucleation intensified in distinct interfacial domains with high nucleation flux, indicated by red and orange areas. Increased supersaturation ratio led to an overall larger number of nuclei being formed per unit area throughout the LC-STEM experiment with higher local nucleation fluxes occurring earlier in the time-lapsed data (Figure 2b). At higher magnification (Figure 2c), almost all nuclei formed with high flux before $30 \mathrm{~s}$, after which nucleation greatly slowed down. There are two noteworthy aspects of the maps. First, the nucleation flux maps showed interfacial domains with locally high nucleation flux (e.g. Figure $\mathbf{2 b}, \mathrm{t}=41 \mathrm{~s}$ ), as opposed to randomly distributed nucleation events on the surface. These interfacial domains of high local nucleation flux appeared in different and exclusive regions of the interface at different times during nucleation. Second, after the initial large nucleation flux at the highest supersaturation ratio, almost no nucleation events were observed after 30 seconds. Together, these two features strongly suggest that nucleation was heterogeneous and occurred at a limited number of nucleation sites.

To investigate the spatial variations in nucleation kinetics in more detail, static nucleation kinetic maps were generated by correlating nucleation induction time and nanoparticle spatial location. Briefly, these maps were generated by interpolating a 3D surface of the nucleation induction time given known values for each nanocrystal observed in the LC-STEM images (see Supporting information for methods). Each map derives from a single LC-STEM movie taken at a given experimental condition and shows a static representation of the local nucleation kinetics at the same spatial scale as the LC-STEM images. Figure 3 shows nanometric spatial maps of nucleation kinetics for three different image magnifications and two different precursor 
concentrations. The blue dots in each map represent positions of silver nanocrystals extracted from the last frame of the LC-STEM movie, while the heat map is the interpolated nucleation induction time. Yellow/white areas represent domains where nucleation happened late in the movie, i.e. slow nucleation, and black/red areas indicate where nucleation occurred early in the movie, i.e. fast nucleation. At the lowest relative supersaturation ratio $(0.1 \mathrm{mM}, 80 \mathrm{kx})$, the nucleation map was dominated by slow nucleation regions and interspersed with a few red/black $100-200 \mathrm{~nm}$ regions showing more rapid nucleation. As the supersaturation was increased by increasing LC-STEM magnification $(0.1 \mathrm{mM}, 100 \mathrm{kx})$, the nucleation map showed increased coverage of $100-200 \mathrm{~nm}$ red and black fast nucleation domains. We note that each domain contained at least $5-10$ nanocrystals that nucleated within a few seconds of each other, indicating the appearance of domains was not an artifact of the map interpolation procedure. The nucleation map for the highest supersaturation ratio for $0.1 \mathrm{mM}$ precursor $(150 \mathrm{kx})$ showed rapid nucleation kinetics over much of the area with only a few single particles showing slow nucleation. We observed the same qualitative trend for the $0.2 \mathrm{mM}$ precursor concentration when increasing supersaturation using the image magnification. For the same magnification, the $0.2 \mathrm{mM}$ precursor concentration experiments showed higher coverage of fast nucleation domains compared to the $0.1 \mathrm{mM}$ precursor concentration. The largest supersaturation ratio of all experiments $(0.2 \mathrm{mM}, 150 \mathrm{kx})$ showed uniformly rapid nucleation kinetics across the map.

Clearly, there was some preference for nanocrystals to nucleate in certain areas on the interface, but what caused the spatial variations in the nucleation kinetics of silver nanocrystals? Additionally, why does increasing supersaturation ratio nearly eliminate spatial variations in nucleation kinetics? Based on currently accepted classical and non-classical nucleation mechanisms, we consider three possible explanations for the observed spatial variations in 
nucleation kinetics of silver nanocrystals. These include random variations in nucleation kinetics, spinodal decomposition, and heterogeneous nucleation. Nucleation is an inherently stochastic process, occurring randomly in time and space even for a constant supersaturation ratio and free energy barrier. It is possible this could lead to perceived spatial non-uniformities in nucleation kinetics. However, nucleation maps in Figure 3 and Figure $\mathbf{S 2}$ rule out this possibility by showing the significant impact of supersaturation on spatial non-uniformities in nucleation kinetics. Nucleation domains located at opposite sides of the map had similar nucleation times despite being separated by nearly a micron (Figure 3b), further indicating the domains were not due to random variations in nucleation but instead were physical in nature.

We have considered spinodal decomposition as a possible mechanism because prior LCTEM experiments by Loh et al. showed evidence for this mechanism during metal nanocrystal nucleation in very thin liquid layers of $\sim 50 \mathrm{~nm} .{ }^{26}$ Spinodal decomposition occurs when the supersaturation ratio is large enough to render the free energy barrier to nucleation insignificant. ${ }^{52}$ Local solute concentration fluctuations cause spontaneous liquid-liquid phase separation to form precursor-rich and solvent-rich phases, followed by nucleation in the precursor rich phase. Two characteristic features of spinodal decomposition are the co-existence of small and large nanocrystals ${ }^{52}$ and a diffusively controlled characteristic time scale for spinodal domain formation. Our LC-STEM images did not show a bimodal population of nanocrystal sizes (Figure 1a-d). The formation time of spinodal regions should scale as $\tau \approx \frac{l^{2}}{D}$, where $D$ is the diffusion coefficient and $l$ is the characteristic length scale. ${ }^{26}$ Taking the characteristic feature size to be the size of the nucleation domains $(l \sim 100 \mathrm{~nm})$ and the diffusion coefficient of silver atoms in liquid to be $D \sim 10^{-9} \frac{\mathrm{m}^{2}}{\mathrm{~s}}$, we obtain a predicted time scale for spinodal domain formation to be $\tau \sim 10^{-5} \mathrm{~s}$, far shorter than the time scale of our experiments. While the patterns observed in our nucleation maps 
are reminiscent of spinodal decomposition, inconsistences between our experiments and theory indicate spinodal decomposition is likely not the underlying mechanism.

An alternative hypothesis is that nanocrystals form with spatially varying nucleation kinetics due to heterogeneous nucleation. Interfacial defects, surface roughness, and surface functional groups decrease the local free energy barrier for nucleation by decreasing the nucleisolvent interfacial energy or locally increasing supersaturation. ${ }^{9}$ To test whether heterogeneous nucleation caused spatial variations in nucleation kinetics, we performed liquid phase atomic force microscopy (AFM) to investigate the properties of the solid/liquid interface. The AFM was operated in chemical force microscopy (CFM) imaging mode to show chemical contrast due to hydrophilic/hydrophobic interactions (see Supporting information for methods). ${ }^{53,54}$ The image in Figure 4a is an AFM height map of the interface and shows the interface was relatively flat with a surface roughness of about $3 \mathrm{~nm}$. However, the phase contrast CFM image (Figure 4b) showed distinct domains with different hydrophilic and hydrophobic nature. Prior work established that attractive hydrophilic tip-surface interactions cause retarded phase shifts (dark contrast) in CFM images. ${ }^{55}$ Moreover, the sizes of the low phase dark domains are on the same scale $(\sim 50-100 \mathrm{~nm})$ as the domains of fast nucleation observed in the nucleation maps in Figure 3.

Silicon nitride surfaces possess silamine $\left(\mathrm{Si}-\mathrm{NH}_{2}\right)$ and silanol $(\mathrm{Si}-\mathrm{OH})$ functional groups, each of which are hydrophilic and may act as heterogeneous nucleation sites. ${ }^{6}$ 9, 10 Prior measurements have found the majority of groups on silicon nitride are silanol, with the fraction of silamine groups present ranging from $1 \%$ to $6 \%{ }^{56,57}$ We performed nanoparticle functional group labeling experiments to probe the spatial distribution of silanol and silamine groups to test our hypothesis that hydrophilic domains are composed of surface functional groups. The silicon nitride membranes were plasma treated in air and then silanized with (3-Aminopropyl)triethoxysilane 
(APTES) to convert all silanol functional groups to amine groups, enabling their facile labeling with amide chemistry. ${ }^{58}$ Amine surface groups created by silanization were labeled with $10 \mathrm{~nm}$ carboxylated gold nanoparticles by reacting 1-ethyl-3-(3-dimethylaminopropyl)carbodiimide hydrochloride (EDC) and N-hydroxysulfosuccinimide (sulfo-NHS) with the carboxyl groups, rendering the nanoparticles reactive to primary amines. Upon incubating the amine reactive nanoparticles with the silicon nitride surface, the nanoparticles covalently bind the amine groups and label the original positions of the silanol groups (see Supporting information for more details). This method also labels native silamine groups, but prior literature and control experiments indicated the silamine coverage was at least five times lower than the total functional group coverage (see Supporting information Figure S5). Figures 4c-d shows TEM images of gold nanoparticles conjugated to a plasma treated silicon nitride membrane surface, denoting the positions of silanol and silamine functional groups. The TEM images clearly show domains of functional groups on the surface with similar sizes as those in the CFM images (Figure 4d) and nucleation kinetic maps (Figure 3). The domains were not observed when the native silamine groups were specifically labeled (Figure 4e) or on the native silicon nitride surface without plasma treatment (Figure S5a). Silicon dioxide surfaces only possess silanol functional groups, so we performed similar labeling experiments on $\mathrm{SiO}_{2}$ membranes to determine whether similar domains formed. We found that silanol groups also formed domains on $\mathrm{SiO}_{2}$ after oxygen plasma treatment (Figure S6), suggesting silanol groups form domains on silicon nitride surfaces, as silicon nitride has similar surface chemistry to $\mathrm{SiO}_{2}$ after exposure to air. ${ }^{59}$ Air plasma generates $\mathrm{O}^{2-}$ and $\mathrm{O}^{-}$ gaseous species that react with the silicon nitride surface to generate additional Si-O groups and polymerize neighboring silanol groups via $\mathrm{Si}-\mathrm{O}-\mathrm{Si}$ siloxane bonds. The polymerized domains become hydrated in water and form domains of silanol groups. ${ }^{60-65}$ Based on our labeling results 
and prior literature, we conclude that oxygen plasma generation of silanol groups and their polymerization formed hydrophilic functional group domains on silicon nitride. Because our labeling method also marks silamine groups, it is possible that a minority fraction of silamine groups exist within the functional group domains.

Silanol and silamine functional groups are pronated or deprotonated depending on $\mathrm{pH}$ and have well-known pKa values. ${ }^{66,67}$ In our LC-STEM experiments, the electron beam modifies the $\mathrm{pH}$ by creating protons and gives a $\mathrm{pH}$ in the range of $5-6 .{ }^{30}$ In this range, $\mathrm{Si}-\mathrm{OH}$ is $50-100 \%$ deprotonated to the form $\mathrm{Si}-\mathrm{O}^{-}$, while $\mathrm{Si}-\mathrm{NH}_{2}$ is $100 \%$ protonated to $\mathrm{Si}_{-} \mathrm{NH}_{3}{ }^{+}$. (see calculations in Supporting information) Prior LC-TEM experiments showing pinning of positively charged gold nanorods during surface diffusion further support the existence of negatively charged, deprotonated silanol groups on the silicon nitride surface. ${ }^{68,69}$ Electrostatic attraction of hydrated $\mathrm{Ag}^{+}$ions to $\mathrm{Si}-\mathrm{O}^{-}$groups could lead to a locally increased supersaturation ratio and nucleation rate on the functional group domains. Alternatively, a decreased interfacial energy penalty for heterogeneous nucleation on the functional group domains could similarly increase nucleation rate. It is currently unclear which phenomena enhances nucleation; however, the latter has a more significant impact on the nucleation rate in the context of CNT, which predicts the free energy barrier for nucleation decreases as the cube of interfacial energy.

\section{Phenomenological nucleation model}

CNT has been shown in multiple instances to quantitatively describe heterogeneous nucleation at solid-liquid interfaces, ${ }^{11}, 14,35$ so we have used CNT as the basis of a phenomenological model to support our experiments. We model the solid/liquid interface as two populations of nucleation sites, one with relatively low free energy barrier (functional group 
domains) and the other population with a higher free energy barrier (surrounding areas). The free energy barrier distribution for each population is taken to be a normal distribution. Based on CNT, the expected heterogeneous nucleation rate for a given nucleation site $(i)$ can be written as ${ }^{70}$

$$
\begin{aligned}
& R_{i}=\operatorname{vexp}\left[-\frac{\Delta G_{h e t, i}}{k_{B} T}\right] \\
& \Delta G_{\text {het }, i}=\phi_{i} \Delta G_{\text {hom }} .
\end{aligned}
$$

Here $v$ is the attempt frequency for nucleation, $\Delta G_{h e t, i}$ is the free energy barrier of nucleation site $i, \Delta G_{\text {hom }}$ is the free energy barrier for homogeneous nucleation, and $\phi_{i}$ is the free energy barrier reduction factor for nucleation site $i$, which depends on the contact angle of the nucleus and is confined to values between $0-1 .{ }^{71} \Delta G_{\text {hom }}$ is determined here using a model derived by Privman et al. for the nucleation of gold nanoparticles in aqueous solution. ${ }^{72}$ Using experimental parameters relevant to our LC-STEM experiments, we estimate the homogeneous nucleation free energy barrier to be $\Delta G_{\text {hom }}=25-40 k_{B} T$ (see Supporting information methods for details). Two populations of nucleation sites are modeled with two normal distributions of free energy barrier reduction factors, $\phi_{i, 1}$ and $\phi_{i, 2}$, which are multiplied with $\Delta G_{h o m}$ to determine the $\Delta G_{\text {het }, i}$ values for a given supersaturation ratio. The average nucleation rate is found by summing over all nucleation rates for a given population of nucleation sites:

$$
\langle R\rangle=v \sum_{i=1}^{N} \exp \left[-\frac{\Delta G_{h e t, i}}{k_{B} T}\right]
$$

Figure 5 shows an example of two populations of nucleation sites used in this model, chosen such that the lowest free energy barrier sampled is $\sim 10 k_{B} T$, consistent with previously established values for heterogeneous nucleation. ${ }^{6}$ We varied the homogeneous free energy barrier between $\Delta G_{\text {hom }}=25-35 k_{B} T\left(S=1.2 \times 10^{7}-1.2 \times 10^{8}\right)$ and calculated the nucleation rate 
for a given site as a function of the free energy barrier reduction factor, $\phi$ (Figure 5b). For the lowest supersaturation ratio (purple line), both populations of nucleation sites have slow nucleation kinetics, consistent with nearly uniform slow nucleation shown in the top left nucleation kinetic map in Figure 3a. In this case, all nucleation sites have high free energy barriers due to the large $\Delta G_{\text {hom }}$ and therefore slow nucleation rates. As supersaturation ratio is increased, nucleation of nearly all the low energy nucleation sites occurs with an appreciable rate and increasing portions of the high energy nucleation sites also have appreciable rates (e.g. red and blue curves, Figure 5b). This condition qualitatively corresponds to the nucleation map in Figure 3b. At the highest supersaturation, nucleation rates for the high and low energy nucleation sites are appreciable and have similar order of magnitude and therefore both nucleate relatively rapidly, consistent with the absence of spatial non-uniformities in the nucleation kinetic map for the highest supersaturation ratio (Figure 3f).

The average nucleation rate, $<\mathrm{R}>$, was calculated for the low and high free energy barrier and all nucleation sites as a function of supersaturation ratio using equation (3), shown in Figure 5c. There is a nearly linear increase in total nucleation rate as a function of supersaturation ratio, consistent with the linear decrease in median nucleation induction time in Figure 1g. As expected from CNT, the slope for the low free energy barrier sites is larger than the high free energy sites. Interestingly, the model predicts that the high free energy barrier site nucleation rate only contributes significantly to the total nucleation rate at high supersaturation levels, which explains the trends in the number of particles vs. time data in Figure 1e. At low LC-STEM magnifications and supersaturation levels, the nucleation rate is dominated by the low free energy barrier nucleation sites and thus only one rate is observed after an initial lag time (e.g. $80 \mathrm{kx}$ and $100 \mathrm{kx}$ data). However, at high magnification and supersaturation ratio, there was an initially high 
nucleation rate followed by a distinct decrease in slope after 20 seconds. In the context of our model, the change point in slope is due to depletion of low free energy barrier nucleation sites, leaving only high free energy sites with a lower nucleation rate.

Quantitative differences between the nucleation kinetic maps in Figure 3 and nanoparticle labeling and CFM images in Figure $\mathbf{4}$ are similarly explained by this model. There exists a hypothetical supersaturation ratio that enables nucleation to occur at an appreciable rate only on the functional group domains with essentially no nucleation elsewhere (e.g. Figure $\mathbf{5 b}$, yellow line), which would lead to a nucleation map that appears identical to nanoparticle labeling images. However, the stochastic nature of nucleation dictates that even for a constant supersaturation and free energy barrier, nucleation will occur sporadically in time. This indicates that not all nuclei will form at the exact same time on the functional group domains, which will create random features in the nucleation maps that are not seen in the nanoparticle labeling experiments.

\section{Conclusions}

We investigated the heterogeneous nucleation of silver nanocrystals at a solid-liquid interface using LC-STEM. Nanoscale mapping of local nucleation kinetics demonstrated nonintuitive spatial variations in heterogeneous nucleation kinetics at a seemingly uniform interface. CFM imaging and functional group labeling experiments revealed that surface functional groups, specifically plasma generated silanol groups, were segregated into interfacial domains that acted as preferential nucleation sites. We derived a phenomenological nucleation model, which was qualitatively consistent with the spatial variations in nucleation kinetics and their dependence on supersaturation ratio. We expect these results will have implications in the broader chemistry community. First, our results illuminate that prior ensemble measurements of nucleation kinetics 
on macroscopically homogeneous and uniform surfaces are an average of the nucleation kinetics at each nucleation site. ${ }^{9,}{ }^{11}$ In reality, microscopic spatial variations in surface chemistry on the macroscopically uniform surface lead to significant spatial variations in nucleation kinetics. As nucleation kinetics often determine crystal polymorph, ${ }^{9}$ these microscopic variations must be considered when investigating morphology of crystals formed at solid-liquid interfaces. Secondly, our results represent a new microscopy and data analysis approach for measuring nanoscale variations in nucleation kinetics on a chemically heterogeneous surface. We expect a similar method could be applied to topologically heterogeneous surfaces as well. This approach could be applied to important materials systems where heterogeneous nucleation occurs at a chemically or topologically non-uniform interface, such as biomineralization, ice nucleation on aerosols, and polymorph selection in templated synthesis of complex oxide materials.

\section{Supporting Information}

Materials and methods; Computational methods for generating nucleation maps; Nucleation induction time maps at different imaging conditions; Functional group labeling control experiments; Functional group protonation calculations; CFM control experiments; Classical nucleation theory calculations.

\section{Acknowledgements}

T.J.W. acknowledges funding from Oak Ridge Associated Universities (ORAU, Award 17061851) and University of Maryland start-up funds. M.W. acknowledges funding from a Harry K. Wells Fellowship from the University of Maryland Energy Innovation Institute. C.P. acknowledges partial funding from Air Force Office of Scientific Research (Grant FA9550- 18-10144). 


\section{References}

1. Bartels-Rausch, T., Ten things we need to know about ice and snow. Nature 2013, 494, 27.

2. Van Driessche, A. E. S.; Van Gerven, N.; Bomans, P. H. H.; Joosten, R. R. M.; Friedrich, H.; GilCarton, D.; Sommerdijk, N. A. J. M.; Sleutel, M., Molecular nucleation mechanisms and control strategies for crystal polymorph selection. Nature 2018, 556, 89.

3. Howes, P. D.; Chandrawati, R.; Stevens, M. M., Colloidal nanoparticles as advanced biological sensors. Science 2014, 346 (6205), 1247390.

4. Dong, Z.; Feng, L.; Hao, Y.; Chen, M.; Gao, M.; Chao, Y.; Zhao, H.; Zhu, W.; Liu, J.; Liang, C.; Zhang, Q.; Liu, Z., Synthesis of Hollow Biomineralized CaCO3-Polydopamine Nanoparticles for Multimodal Imaging-Guided Cancer Photodynamic Therapy with Reduced Skin Photosensitivity. J. Am. Chem. Soc. 2018, 140 (6), 2165-2178.

5. Smeets, P. J. M.; Cho, K. R.; Kempen, R. G. E.; Sommerdijk, N. A. J. M.; De Yoreo, J. J., Calcium carbonate nucleation driven by ion binding in a biomimetic matrix revealed by in situ electron microscopy. Nat. Mater.2015, 14 (4), 394-399.

6. Chayen, N. E.; Saridakis, E.; Sear, R. P., Experiment and theory for heterogeneous nucleation of protein crystals in a porous medium. Proc. Natl. Acad. Sci. U. S. A. 2006, 103 (3), 597-601.

7. Page, A. J.; Sear, R. P., Heterogeneous Nucleation in and out of Pores. Phys. Rev. Lett. 2006, 97 (6), 065701.

8. Diao, Y.; Myerson, A. S.; Hatton, T. A.; Trout, B. L., Surface Design for Controlled Crystallization: The Role of Surface Chemistry and Nanoscale Pores in Heterogeneous Nucleation. Langmuir 2011, 27 (9), 5324-5334.

9. $\quad H u$, Q.; Nielsen, M. H.; Freeman, C. L.; Hamm, L. M.; Tao, J.; Lee, J. R. I.; Han, T. Y. J.; Becker, U.; Harding, J. H.; Dove, P. M.; De Yoreo, J. J., The thermodynamics of calcite nucleation at organic interfaces: Classical vs. non-classical pathways. Faraday Discuss. 2012, 159 (0), 509-523.

10. Aizenberg, J.; Black, A. J.; Whitesides, G. M., Control of crystal nucleation by patterned selfassembled monolayers. Nature 1999, 398 (6727), 495-498.

11. Hamm, L. M.; Giuffre, A. J.; Han, N.; Tao, J.; Wang, D.; De Yoreo, J. J.; Dove, P. M., Reconciling disparate views of template-directed nucleation through measurement of calcite nucleation kinetics and binding energies. Proc. Natl. Acad. Sci. 2014, 111 (4), 1304-1309.

12. Sear, R. P., Quantitative studies of crystal nucleation at constant supersaturation: experimental data and models. CrystEngComm 2014, 16 (29), 6506-6522.

13. Yau, S. T.; Vekilov, P. G., Direct Observation of Nucleus Structure and Nucleation Pathways in Apoferritin Crystallization. J. Am. Chem. Soc. 2001, 123 (6), 1080-1089.

14. Sleutel, M.; Lutsko, J.; Van Driessche, A. E. S.; Durán-Olivencia, M. A.; Maes, D., Observing classical nucleation theory at work by monitoring phase transitions with molecular precision. Nat. Commun. 2014, 5, 5598.

15. Chen, J.; Zhu, E.; Liu, J.; Zhang, S.; Lin, Z.; Duan, X.; Heinz, H.; Huang, Y.; De Yoreo, J. J., Building two-dimensional materials one row at a time: Avoiding the nucleation barrier. Science 2018, 362 (6419), 1135-1139.

16. Ma, X.; Zhang, S.; Jiao, F.; Newcomb, C. J.; Zhang, Y.; Prakash, A.; Liao, Z.; Baer, M. D.; Mundy, C. J.; Pfaendtner, J.; Noy, A.; Chen, C.-L.; De Yoreo, J. J., Tuning crystallization pathways through sequence engineering of biomimetic polymers. Nat. Mater. 2017, 16, 767.

17. Liu, G.; Liu, J.; Sun, H.; Zheng, X.; Liu, Y.; Li, X.; Qi, H.; Bai, X.; Jackson, K. A.; Tao, X., In Situ Imaging of On-Surface, Solvent-Free Molecular Single-Crystal Growth. J. Am. Chem. Soc. 2015, 137 (15), 49724975. 
18. Lupulescu, A. I.; Rimer, J. D., In Situ Imaging of Silicalite-1 Surface Growth Reveals the Mechanism of Crystallization. Science 2014, 344 (6185), 729-732.

19. Zheng, H.; Smith, R. K.; Jun, Y.-w.; Kisielowski, C.; Dahmen, U.; Alivisatos, A. P., Observation of Single Colloidal Platinum Nanocrystal Growth Trajectories. Science 2009, 324 (5932), 1309-1312.

20. Woehl, T. J.; Park, C.; Evans, J. E.; Arslan, I.; Ristenpart, W. D.; Browning, N. D., Direct Observation of Aggregative Nanoparticle Growth: Kinetic Modeling of the Size Distribution and Growth Rate. Nano Lett. 2014, 14 (1), 373-378.

21. Jungjohann, K. L.; Bliznakov, S.; Sutter, P. W.; Stach, E. A.; Sutter, E. A., In Situ Liquid Cell Electron Microscopy of the Solution Growth of Au-Pd Core-Shell Nanostructures. Nano Lett. 2013, 13 (6), 29642970.

22. Yuk, J. M.; Park, J.; Ercius, P.; Kim, K.; Hellebusch, D. J.; Crommie, M. F.; Lee, J. Y.; Zettl, A.; Alivisatos, A. P., High-Resolution EM of Colloidal Nanocrystal Growth Using Graphene Liquid Cells. Science 2012, 336 (6077), 61-64.

23. Hauwiller, M. R.; Ondry, J. C.; Chan, C. M.; Khandekar, P.; Yu, J.; Alivisatos, A. P., Gold Nanocrystal Etching as a Means of Probing the Dynamic Chemical Environment in Graphene Liquid Cell Electron Microscopy. J. Am. Chem. Soc. 2019, 141 (10), 4428-4437.

24. Wu, J.; Gao, W.; Yang, H.; Zuo, J.-M., Dissolution Kinetics of Oxidative Etching of Cubic and Icosahedral Platinum Nanoparticles Revealed by in Situ Liquid Transmission Electron Microscopy. ACS Nano 2017, 11 (2), 1696-1703.

25. Hauwiller, M. R.; Frechette, L. B.; Jones, M. R.; Ondry, J. C.; Rotskoff, G. M.; Geissler, P.; Alivisatos, A. P., Unraveling Kinetically-Driven Mechanisms of Gold Nanocrystal Shape Transformations Using Graphene Liquid Cell Electron Microscopy. Nano Lett. 2018, 18 (9), 5731-5737.

26. Loh, N. D.; Sen, S.; Bosman, M.; Tan, S. F.; Zhong, J.; Nijhuis, C. A.; Král, P.; Matsudaira, P.; Mirsaidov, U., Multistep nucleation of nanocrystals in aqueous solution. Nat. Chem. 2016, 9, 77.

27. Wang, M.; Park, C.; Woehl, T. J., Quantifying the Nucleation and Growth Kinetics of Electron Beam Nanochemistry with Liquid Cell Scanning Transmission Electron Microscopy. Chem. Mater. 2018, 30 (21), 7727-7736.

28. Woehl, T. J.; Evans, J. E.; Arslan, I.; Ristenpart, W. D.; Browning, N. D., Direct in Situ Determination of the Mechanisms Controlling Nanoparticle Nucleation and Growth. ACS Nano 2012, 6 (10), 8599-8610.

29. WOEHL, T. J.; ABELLAN, P., Defining the radiation chemistry during liquid cell electron microscopy to enable visualization of nanomaterial growth and degradation dynamics. J. Microsc. 2017, 265 (2), 135147.

30. Schneider, N. M.; Norton, M. M.; Mendel, B. J.; Grogan, J. M.; Ross, F. M.; Bau, H. H., ElectronWater Interactions and Implications for Liquid Cell Electron Microscopy. J. Phys. Chem. C 2014, 118 (38), 22373-22382.

31. Park, J. H.; Schneider, N. M.; Grogan, J. M.; Reuter, M. C.; Bau, H. H.; Kodambaka, S.; Ross, F. M., Control of Electron Beam-Induced Au Nanocrystal Growth Kinetics through Solution Chemistry. Nano Lett. 2015, 15 (8), 5314-5320.

32. Grogan, J. M.; Schneider, N. M.; Ross, F. M.; Bau, H. H., Bubble and Pattern Formation in Liquid Induced by an Electron Beam. Nano Lett. 2014, 14 (1), 359-364.

33. Moser, T. H.; Mehta, H.; Park, C.; Kelly, R. T.; Shokuhfar, T.; Evans, J. E., The role of electron irradiation history in liquid cell transmission electron microscopy. $\quad$ Sci. Adv. 2018, 4 (4), eaaq1202.

34. Jun, Y.-S.; Kim, D.; Neil, C. W., Heterogeneous Nucleation and Growth of Nanoparticles at Environmental Interfaces. Acc. Chem. Res. 2016, 49 (9), 1681-1690.

35. Li, Q.; Jun, Y.-S., The apparent activation energy and pre-exponential kinetic factor for heterogeneous calcium carbonate nucleation on quartz.

Commun. Chem. 2018, 1 (1), 56. 
36. Smeets, P. J. M.; Finney, A. R.; Habraken, W. J. E. M.; Nudelman, F.; Friedrich, H.; Laven, J.; De Yoreo, J. J.; Rodger, P. M.; Sommerdijk, N. A. J. M., A classical view on nonclassical nucleation. Proc. Natl. Acad. Sci. 2017, 114 (38), E7882-E7890.

37. De Yoreo, J. J.; Vekilov, P. G., Principles of crystal nucleation and growth. Rev. Mineral. Geochem. 2003, 54, 57-93.

38. Zhang, X.; Zhang, F.; Wang, Y.; Schulman, D. S.; Zhang, T.; Bansal, A.; Alem, N.; Das, S.; Crespi, V. H.; Terrones, M.; Redwing, J. M., Defect-Controlled Nucleation and Orientation of WSe2 on hBN: A Route to Single-Crystal Epitaxial Monolayers. ACS Nano 2019, 13 (3), 3341-3352.

39. Fenton, J. L.; Steimle, B. C.; Schaak, R. E., Tunable intraparticle frameworks for creating complex heterostructured nanoparticle libraries. Science 2018, 360 (6388), 513-517.

40. Ding, K.; Cullen, D. A.; Zhang, L.; Cao, Z.; Roy, A. D.; Ivanov, I. N.; Cao, D., A general synthesis approach for supported bimetallic nanoparticles via surface inorganometallic chemistry. Science 2018, 362 (6414), 560-564.

41. Wong, A.; Liu, Q.; Griffin, S.; Nicholls, A.; Regalbuto, J. R., Synthesis of ultrasmall, homogeneously alloyed, bimetallic nanoparticles on silica supports. Science 2017, eaao6538.

42. Fu, Y.; Zheng, W.; Wang, X.; Hautzinger, M. P.; Pan, D.; Dang, L.; Wright, J. C.; Pan, A.; Jin, S., Multicolor Heterostructures of Two-Dimensional Layered Halide Perovskites that Show Interlayer Energy Transfer. J. Am. Chem. Soc. 2018, 140 (46), 15675-15683.

43. Van Vleet, M. J.; Weng, T.; Li, X.; Schmidt, J. R., In Situ, Time-Resolved, and Mechanistic Studies of Metal-Organic Framework Nucleation and Growth. Chem. Rev. 2018, 118 (7), 3681-3721.

44. Wang, X.-G.; Cheng, Q.; Yu, Y.; Zhang, X.-Z., Controlled Nucleation and Controlled Growth for Size Predicable Synthesis of Nanoscale Metal-Organic Frameworks (MOFs): A General and Scalable Approach. Angew. Chem., Int. Ed. 2018, 57 (26), 7836-7840.

45. Lan, X.; Huang, N.; Wang, J.; Wang, T., A general and facile strategy for precisely controlling the crystal size of monodispersed metal-organic frameworks via separating the nucleation and growth. Chem. Commun. 2018, 54 (6), 584-587.

46. Udayabhaskararao, T.; Kazes, M.; Houben, L.; Lin, H.; Oron, D., Nucleation, Growth, and Structural Transformations of Perovskite Nanocrystals. Chem. Mater. 2017, 29 (3), 1302-1308.

47. Yao, Z.; Jones, T. W.; Grigore, M.; Duffy, N. W.; Anderson, K. F.; Dunbar, R. B.; Feron, K.; Hao, F.; Lin, H.; Wilson, G. J., Tunable Crystallization and Nucleation of Planar CH3NH3Pbl3 through SolventModified Interdiffusion. ACS Appl. Mater. Interfaces 2018, 10 (17), 14673-14683.

48. Cao, X.; Zhi, L.; Jia, Y.; Li, Y.; Zhao, K.; Cui, X.; Ci, L.; Zhuang, D.; Wei, J., A Review of the Role of Solvents in Formation of High-Quality Solution-Processed Perovskite Films. ACS Appl. Mater. Interfaces 2019, 11 (8), 7639-7654.

49. Lee, J. W.; Yu, H.; Lee, K.; Bae, S.; Kim, J.; Han, G. R.; Hwang, D.; Kim, S. K.; Jang, J., Highly Crystalline Perovskite-Based Photovoltaics via Two-Dimensional Liquid Cage Annealing Strategy. J. Am. Chem. Soc. 2019, 141 (14), 5808-5814.

50. Wang, Y.; Li, L.; Liang, H.; Xing, Y.; Yan, L.; Dai, P.; Gu, X.; Zhao, G.; Zhao, X., Superstructure of a Metal-Organic Framework Derived from Microdroplet Flow Reaction: An Intermediate State of Crystallization by Particle Attachment. ACS Nano 2019, 13 (3), 2901-2912.

51. van Embden, J.; Sader, J. E.; Davidson, M.; Mulvaney, P., Evolution of Colloidal Nanocrystals: Theory and Modeling of their Nucleation and Growth. J. Phys. Chem. C 2009, 113 (37), 16342-16355.

52. Wallace, A. F.; Hedges, L. O.; Fernandez-Martinez, A.; Raiteri, P.; Gale, J. D.; Waychunas, G. A.; Whitelam, S.; Banfield, J. F.; De Yoreo, J. J., Microscopic Evidence for Liquid-Liquid Separation in Supersaturated $\mathrm{CaCO}<\mathrm{sub}>3</$ sub $>$ Solutions. Science 2013, 341 (6148), 885-889.

53. Noy, A.; Sanders, C. H.; Vezenov, D. V.; Wong, S. S.; Lieber, C. M., Chemically-Sensitive Imaging in Tapping Mode by Chemical Force Microscopy: Relationship between Phase Lag and Adhesion. Langmuir 1998, 14 (7), 1508-1511. 
54. Ashby, P. D.; Lieber, C. M., Ultra-Sensitive Imaging and Interfacial Analysis of Patterned Hydrophilic SAM Surfaces Using Energy Dissipation Chemical Force Microscopy. J. Am. Chem. Soc. 2005, 127 (18), 6814-6818.

55. Finot, M. O.; McDermott, M. T., High-Resolution Chemical Mapping of Surface Bound Functional Groups with Tapping-Mode Scanning Force Microscopy. J. Am. Chem. Soc. 1997, 119 (36), 8564-8565.

56. Bousse, L.; Mostarshed, S., The zeta potential of silicon nitride thin films. J. Electroanal. Chem. 1991, 302 (1), 269-274.

57. Guan, W.; Rajan, N. K.; Duan, X.; Reed, M. A., Quantitative probing of surface charges at dielectricelectrolyte interfaces. Lab Chip 2013, 13 (7), 1431-1436.

58. Robertson, A. W.; Zhu, G.; Mehdi, B. L.; Jacobs, R. M. J.; De Yoreo, J.; Browning, N. D., Nanoparticle Immobilization for Controllable Experiments in Liquid-Cell Transmission Electron Microscopy. ACS Appl. Mater. Interfaces 2018, 10 (26), 22801-22808.

59. Kennedy, G. P.; Buiu, O.; Taylor, S., Oxidation of silicon nitride films in an oxygen plasma. J. Appl. Phys 1999, 85 (6), 3319-3326.

60. Zhuravlev, L. T.; Potapov, V. V., Density of silanol groups on the surface of silica precipitated from a hydrothermal solution. Russ. J. Phys. Chem. 2006, 80 (7), 1119-1128.

61. Zhuravlev, L. T., The surface chemistry of amorphous silica. Zhuravlev model. Colloids Surf., $A$ 2000, $173(1), 1-38$.

62. Jiang, L.; Li, S.; Wang, J.; Yang, L.; Sun, Q.; Li, Z., Surface Wettability of Oxygen Plasma Treated Porous Silicon. J. Nanomater. 2014, 2014, 6.

63. Suni, T.; Henttinen, K.; Suni, I.; Mäkinen, J., Effects of Plasma Activation on Hydrophilic Bonding of Si and SiO2. J. Electrochem. Soc. 2002, 149 (6), G348-G351.

64. Kaya, S.; Rajan, P.; Dasari, H.; Ingram, D. C.; Jadwisienczak, W.; Rahman, F., A Systematic Study of Plasma Activation of Silicon Surfaces for Self Assembly. ACS Appl. Mater. Interfaces 2015, 7 (45), 2502425031.

65. Sneh, O.; George, S. M., Thermal Stability of Hydroxyl Groups on a Well-Defined Silica Surface. J. Phys. Chem. 1995, 99 (13), 4639-4647.

66. Cloarec, J.-P.; Chevalier, C.; Genest, J.; Beauvais, J.; Chamas, H.; Chevolot, Y.; Baron, T.; Souifi, A., $\mathrm{pH}$ driven addressing of silicon nanowires onto Si3N4/SiO2micro-patterned surfaces. Nanotechnology 2016, 27 (29), 295602.

67. Tsukruk, V. V.; Bliznyuk, V. N., Adhesive and Friction Forces between Chemically Modified Silicon and Silicon Nitride Surfaces. Langmuir 1998, 14 (2), 446-455.

68. Chee, S. W.; Anand, U.; Bisht, G.; Tan, S. F.; Mirsaidov, U., Direct Observations of the Rotation and Translation of Anisotropic Nanoparticles Adsorbed at a Liquid-Solid Interface. Nano Lett. 2019, 19 (5), 2871-2878.

69. Chee, S. W.; Baraissov, Z.; Loh, N. D.; Matsudaira, P. T.; Mirsaidov, U., Desorption-Mediated Motion of Nanoparticles at the Liquid-Solid Interface. J. Phys. Chem. C 2016, 120 (36), 20462-20470.

70. Sear, R. P.; Howard, M., Modeling dual pathways for the metazoan spindle assembly checkpoint. Proc. Natl. Acad. Sci. 2006, 103 (45), 16758-16763.

71. Thanh, N. T. K.; Maclean, N.; Mahiddine, S., Mechanisms of Nucleation and Growth of Nanoparticles in Solution. Chem. Rev. 2014, 114 (15), 7610-7630.

72. Robb, D. T.; Privman, V., Model of Nanocrystal Formation in Solution by Burst Nucleation and Diffusional Growth. Langmuir 2008, 24 (1), 26-35. 


\section{Figures}
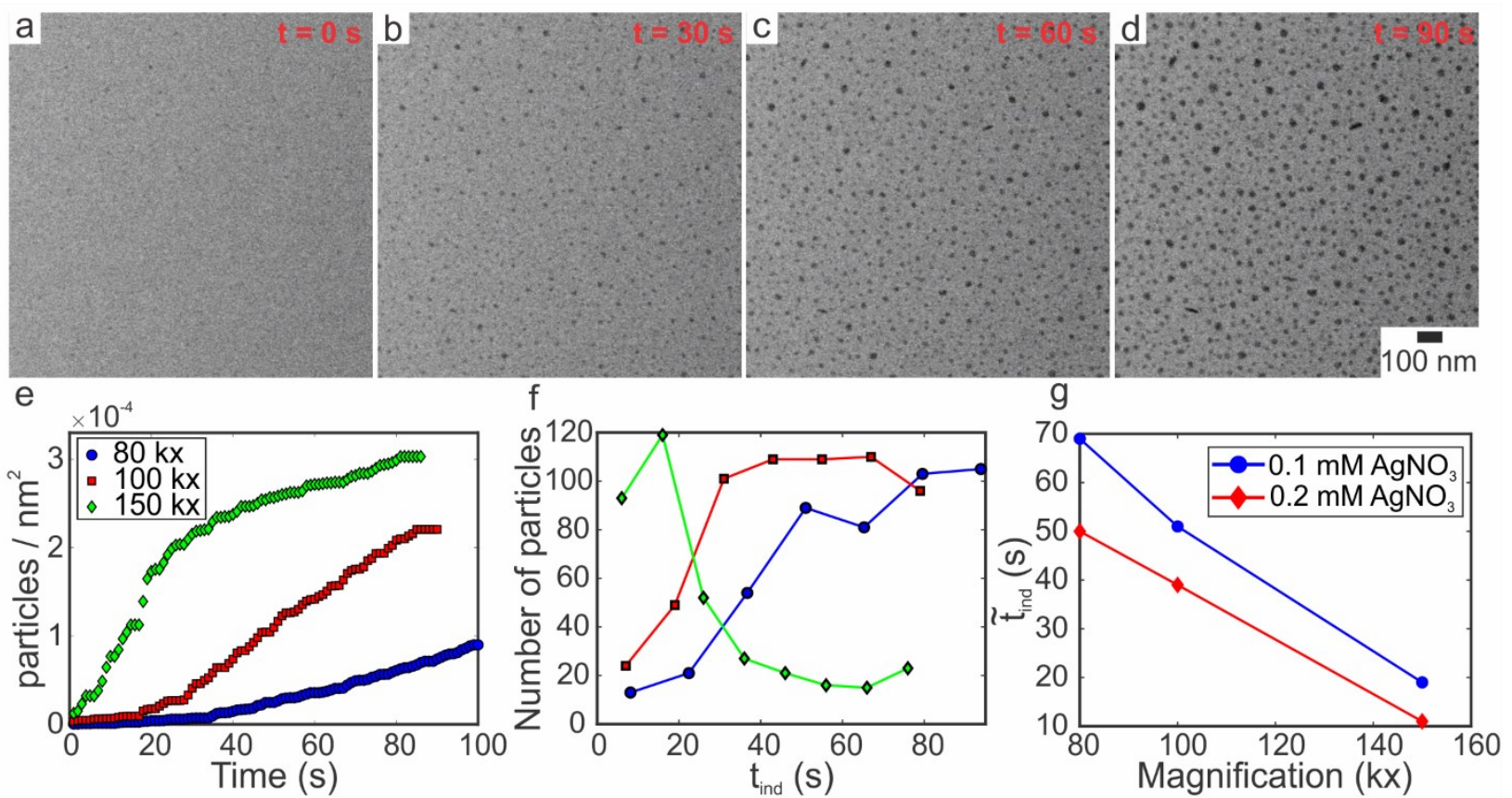

Figure 1. (a-d) Time lapsed bright field STEM images of silver nucleation for an image magnification of $100 \mathrm{kx}$ and precursor concentration of $0.1 \mathrm{mM} \mathrm{AgNO}_{3}$ (beam current $\left(i_{e}\right)$ of $i_{e}=$ $31 \mathrm{pA}$, dose rate $(\dot{d})$ of $\dot{d}=1.3 \mathrm{MGy} / \mathrm{s})$ e) Number of particles per area as a function of time and magnification. f) Distribution of nucleation induction times as a function of magnification, and $\mathrm{g}$ ) Median nucleation induction time as a function of magnification and precursor concentration. 


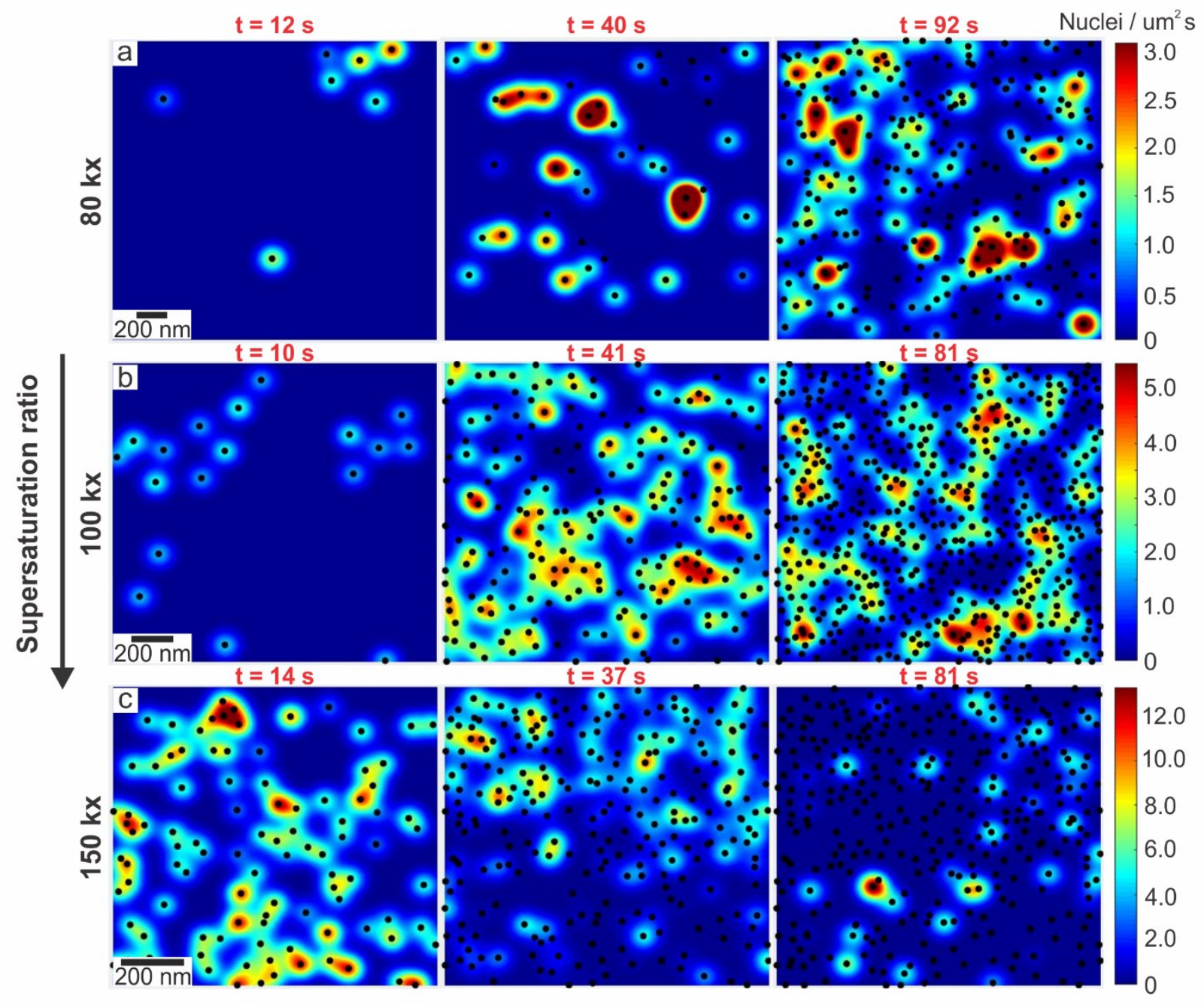

Figure 2. Time-lapsed nucleation flux maps for a precursor concentration of $0.1 \mathrm{mM} \mathrm{AgNO}_{3}$ and LC-STEM magnifications of a) $\left.80 \mathrm{kx}\left(i_{e}=31 \mathrm{pA}, \dot{d}=0.7 \mathrm{MGy} / \mathrm{s}\right), \mathrm{b}\right) 100 \mathrm{kx}\left(i_{e}=31 \mathrm{pA}, \dot{d}=1.3\right.$ $\mathrm{MGy} / \mathrm{s})$, and c) $150 \mathrm{kx}\left(i_{e}=31 \mathrm{pA}, \dot{d}=3.0 \mathrm{MGy} / \mathrm{s}\right)$. The black dots are centroids of silver nanocrystals formed during each LC-STEM experiment, while the color map corresponds to the interpolated nuclei flux. Red and orange areas correspond to high flux (higher nucleation rate), and blue and teal areas represent low flux (lower nucleation rate). 


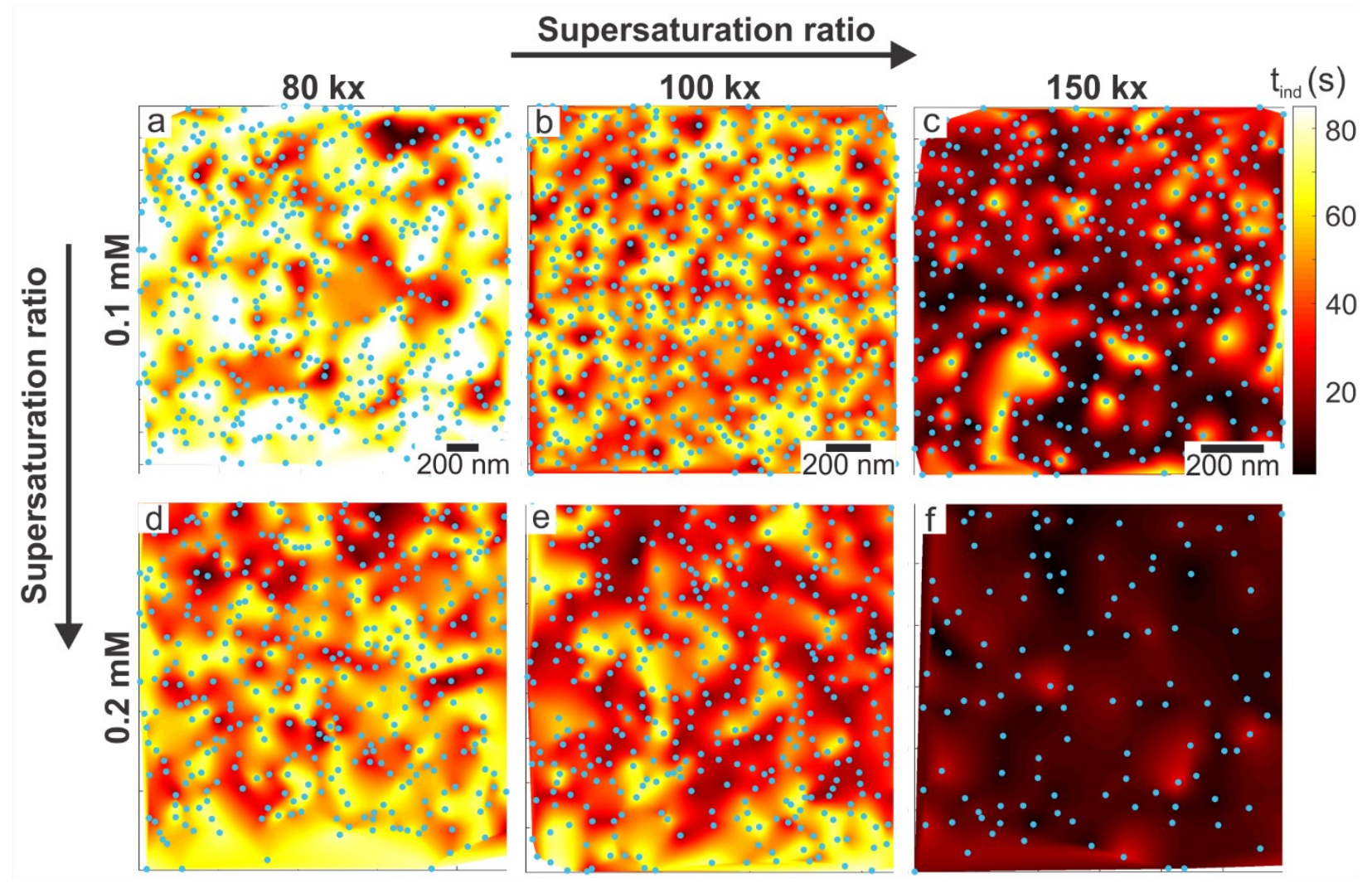

Figure 3. Static nanometric spatial maps of silver nucleation induction times as a function of LCSTEM magnification and precursor concentration. The beam current for all experiments was 31 pA. Top row: $0.1 \mathrm{mM} \mathrm{AgNO}_{3}$ at magnifications of a) $80 \mathrm{kx}(\dot{d}=0.7 \mathrm{MGy} / \mathrm{s})$, b) $100 \mathrm{kx}(\dot{d}=1.3$ $\mathrm{MGy} / \mathrm{s})$, and c) $150 \mathrm{kx}(\dot{d}=3.0 \mathrm{MGy} / \mathrm{s})$. Bottom row: $0.2 \mathrm{mM} \mathrm{AgNO}_{3}$ at magnifications of d) 80 $\mathrm{kx}$, e) $100 \mathrm{kx}$, and f) $150 \mathrm{kx}$. The blue dots are centroids of all silver nanocrystals formed during each LC-STEM experiment, while the color map corresponds to the interpolated local nucleation induction time. Yellow and white areas correspond to large nucleation induction times (low nucleation rate) while red and black are small nucleation induction times (high nucleation rate). 

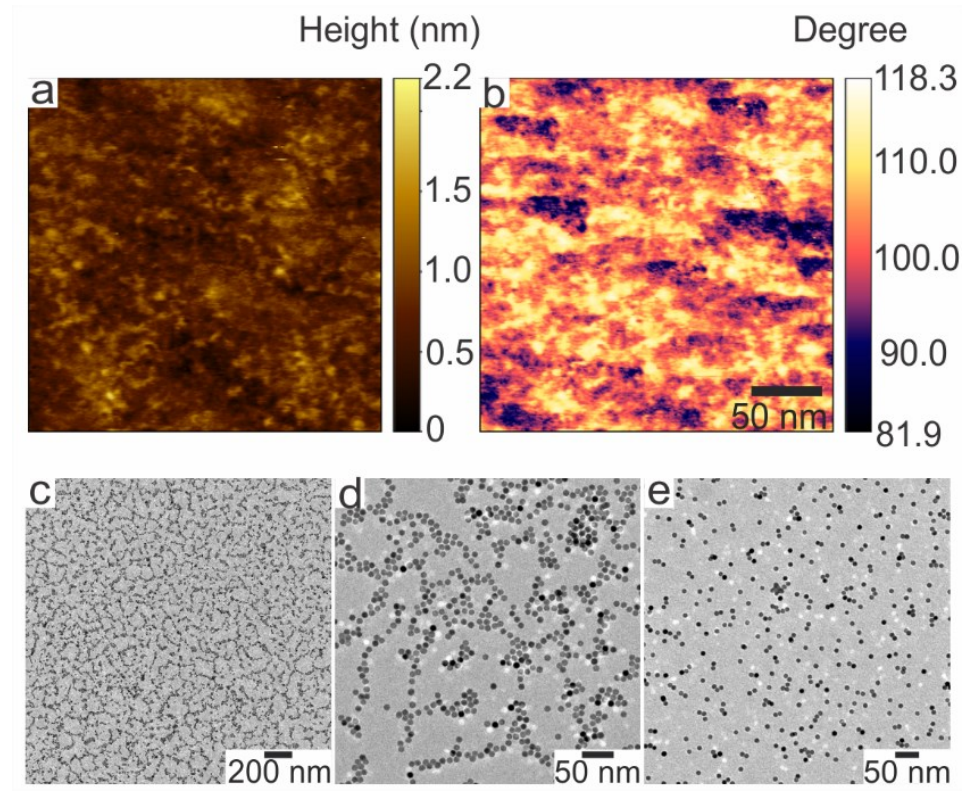

Figure 4. a)-b) Liquid phase AFM and CFM of a plasma treated silicon nitride membrane. a) Tapping mode AFM height image, b) tapping mode CFM phase image. c)-d) Low and high magnification TEM images of gold nanoparticle labeled functional groups (silanol and silamine) on a plasma treated silicon nitride membrane. e) TEM image of gold nanoparticles labeling only the native silamine groups on a plasma treated silicon nitride membrane. 

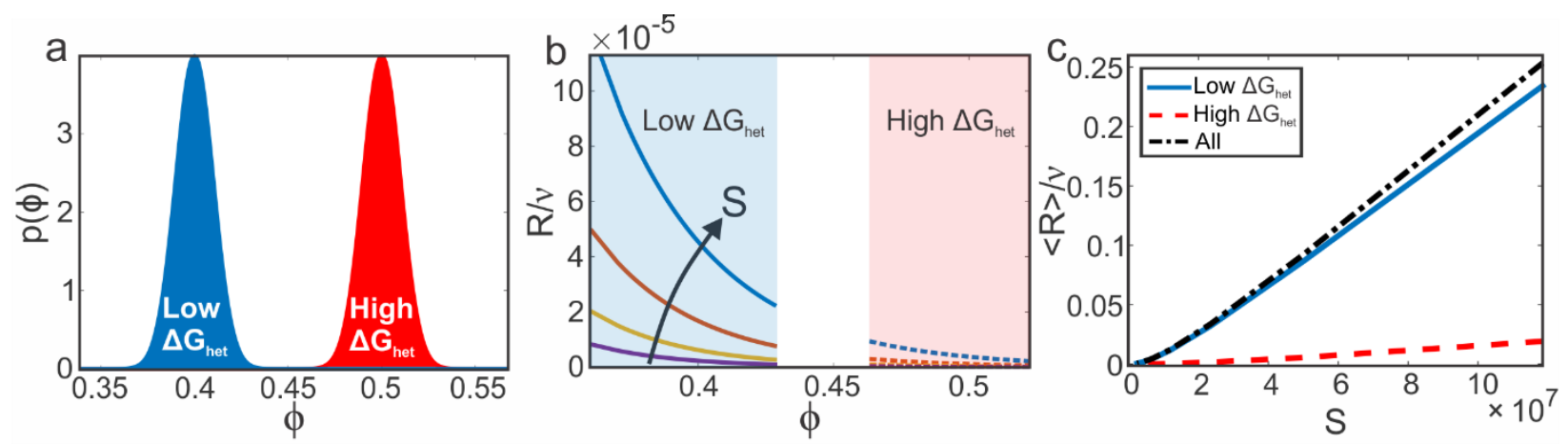

Figure 5. Phenomenological nucleation model. (a) Two populations of nucleation sites with low and high free energy barrier are modeled by two normally distributed probability distribution functions (PDFs) for nucleation site free energy barrier reduction factor, $p(\phi)$. (b) Normalized nucleation rate as a function of free energy barrier reduction factor and supersaturation ratio, calculated from equation (2). Solid lines were calculated by sampling values from the low $\Delta G_{\text {het }}$ PDF (blue PDF in (a)), while dashed lines were sampled from the high $\Delta G_{\text {het }}$ PDF (red PDF in (a)). (c) Average normalized nucleation rates calculated from equation (3) for low and high $\Delta G_{\text {het }}$ nucleation sites and all nucleation sites, as a function of supersaturation ratio. 
TOC Figure

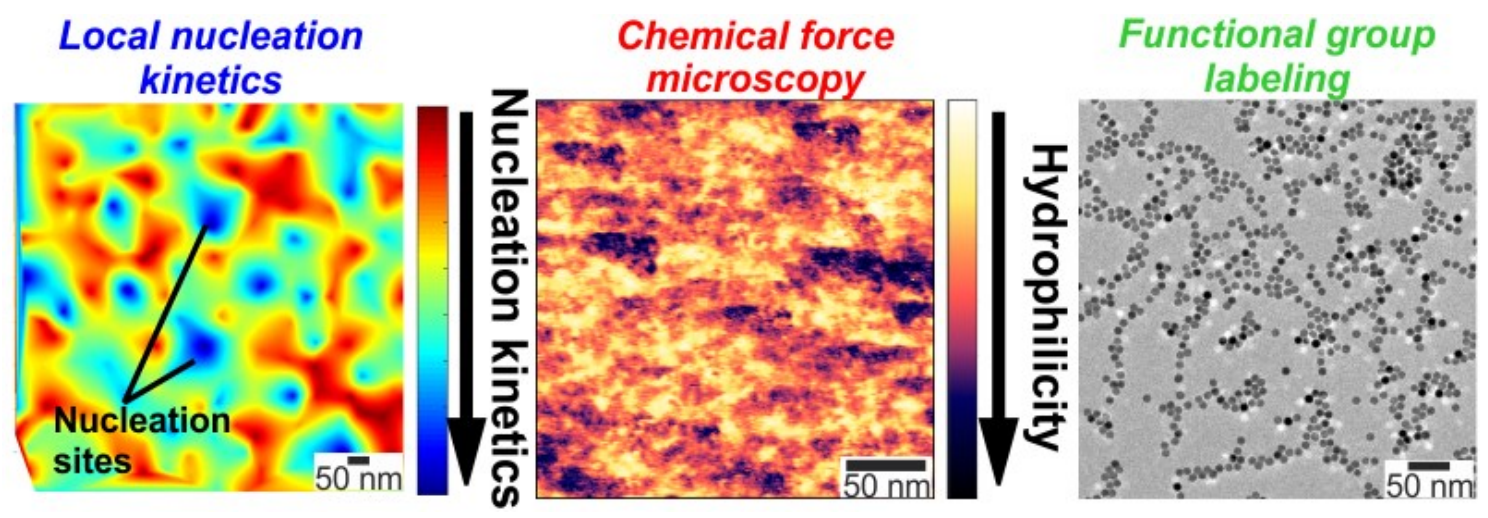


Supporting Information

Nanoscale mapping of non-uniform heterogeneous nucleation kinetics mediated by surface chemistry

Mei Wang, ${ }^{1}$ Thilini U. Dissanayake ${ }^{1}$, Chiwoo Park, ${ }^{2}$ Karen Gaskell, ${ }^{3}$ Taylor J. Woehl ${ }^{1, *}$

${ }^{1}$ Department of Chemical and Biomolecular Engineering, University of Maryland, College Park, Maryland 20742, United States

${ }^{2}$ Department of Industrial and Manufacturing Engineering, Florida State University, Tallahassee, Florida 32306, United States

${ }^{3}$ Department of Chemistry \& Biochemistry, University of Maryland, College Park, Maryland 20742, United States 


\section{Outline}

1. In-situ silver nanoparticle nucleation experiments

2. Multi-particle tracking algorithm

3. Method for generating the nucleation flux map

4. Nanometric spatial map of nucleation induction time

5. Silanization treatment and EDC-NHS coupling

6. Nanoparticle surface labeling control experiments

7. Calculations of functional groups on silicon nitride membrane

8. Atomic force microscopy experimental methods and control experiments 


\section{In-situ silver nanoparticle nucleation experiments.}

The detailed methods for the liquid cell experiments are given elsewhere. ${ }^{1}$ Briefly, the experiments were performed on a JEOL JEM-2100F TEM operating at $200 \mathrm{kV}$ in STEM mode. Liquid cell samples were prepared with a Protochips liquid cell holder (Poseidon Select). The aqueous precursor contained either $0.1 \mathrm{mM} \mathrm{AgNO}_{3}$ or $0.2 \mathrm{mM} \mathrm{AgNO}_{3}$ and $0.1 \mathrm{M}$ tert-butanol and was purged with argon gas for 1 hour prior to experiments. The silicon nitride membranes were plasma cleaned in air for 3 minutes to make the surface hydrophilic (Harrick Plasma, PDC-32G, Max Power $150 \mathrm{~W}$ ). The liquid thickness was around $900 \mathrm{~nm}$ as determined by electron energy loss spectroscopy (EELS). The precursor solution was supplied prior to each nanoparticle nucleation experiment for 5 minutes at a flow rate of $300 \mu \mathrm{L} / \mathrm{h}$ (Harvard Apparatus). LC-STEM movies were recorded with Camtasia Studios. Nucleation experiments were carried out at beam currents of 21 and $31 \mathrm{pA}$ and imaging magnifications of $80 \mathrm{kx}, 100 \mathrm{kx}$, and $150 \mathrm{kx}$. The dwell time at each pixel was $5 \mu$ s and the scanning image size was $1024 \times 1024$ pixel, giving image area averaged dose rates around $10^{6} \mathrm{~Gy} / \mathrm{s}$ (exact values given in figure captions). Dose rates were calculated using the method of Alloyeau et al. ${ }^{2}$

\section{Multi-particle tracking algorithm}

A custom multi-particle tracking algorithm was applied to LC-STEM videos to automatically track individual silver nanocrystals in every frame. ${ }^{3,4}$ The pixel location and area of each particle $i$ observed over time is stored in a matrix in the form of $\left\{\left(\mathrm{x}_{n}^{(\mathrm{i}, \mathrm{t})}, \mathrm{y}_{n}^{(\mathrm{i}, \mathrm{t})}\right) ; n=\right.$ $\left.1, \ldots, N_{i t}\right\}$.The first non-zero time coordinate for each particle represents the nucleation induction time.

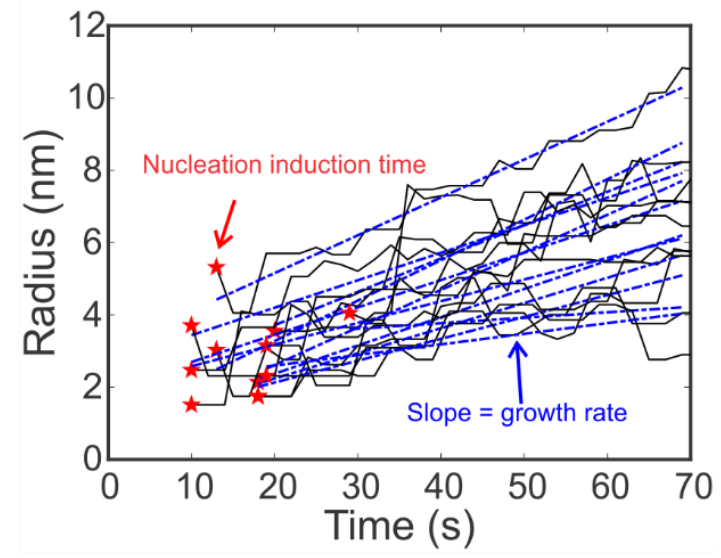


Figure S1. An example of Multi-particle tracking algorithm. Each black line is a single particle growth track in a LC-STEM movie. The first time each particle is detected by the algorithm is equal to its nucleation induction time.

\section{Method for generating the nucleation flux map}

We analyzed Video S1 - S3 using multi-target tracking analysis ${ }^{4}$ to identify nucleation events in the videos. For each of the videos, the nucleation time of a particle is defined by the first appearance time of the particle in the video and the nucleation location is the mass center of the particle at the nucleation time. The nucleation times and locations were recorded as data to estimate the nucleation density map. Let $\left\{\left(t_{i}, \boldsymbol{x}_{i}\right): i=1, \ldots, N\right\}$ denote the $N$ pairs of the times and locations extracted from one of the videos. We consider the data as random events drawn from an inhomogeneous spatiotemporal Poisson process with mean spatiotemporal event intensity function $\lambda(t, x)$. Briefly, a Poisson process models a series of discrete random events occurring over time, nucleation in this case, where there is knowledge of the average time in between the events. This process model assumes the events, nucleation in this case, are memoryless, which applies well to nucleation where a nucleation event has no impact on the timing of future subsequent nucleation events. The mean intensity function practically implies the expected number of nucleation events occurring per unit time and unit area at time $t$ and location $\mathbf{x}$. We refer to this function as the "local nucleation flux" in the manuscript. The unknown intensity function was statistically fit to data $\left\{\left(t_{i}, \boldsymbol{x}_{i}\right): i=1, \ldots, N\right\}$ following a $\log$ Gaussian Cox model. ${ }^{5}$ The fitted intensity function $\lambda(t, \mathbf{x})$ was displayed as an output video (Supplementary Movies S4-S6) to represent a nucleation flux map and its change in time and space (Figure 2 in main text).

\section{Nanometric spatial map of nucleation induction time}

To create the static nucleation kinetic maps (Figure 3 in main text), the centroid of each particle $i$ in the last frame of a LC-STEM video was plotted with MATLAB. A 3D surface of the nucleation induction time was then fit to the centroids of the nanoparticles using natural neighbor interpolation method. ${ }^{6}$ For each of $n$ particle centroids with known values of some function $p\left(x_{i}, y_{i}\right)$ at positions $\left(x_{i}, y_{i}\right)$, two Voronoi diagrams are generated around each position, one where the value of the test position is not included and one where the value of the test position is included. A weight for each neighbor around the test point, $w_{i}$, is calculated by determining how much area 
of a given Voronoi cell is "taken" when the test point is inserted into the Voronoi diagram. The interpolated value at position $(x, y)$ is calculated by

$$
G(x, y)=\sum_{i=1}^{n} w_{i} p\left(x_{i}, y_{i}\right)
$$

To further verify the existence of spatial non-uniformities of local nucleation kinetics, we generated another set of nanometric spatial maps of silver nanocrystal nucleation induction times from LC-STEM movies acquired for a different beam current (Figure S2, beam current 21 pA). These maps showed the same qualitative trends as Figure 3 in the main text.

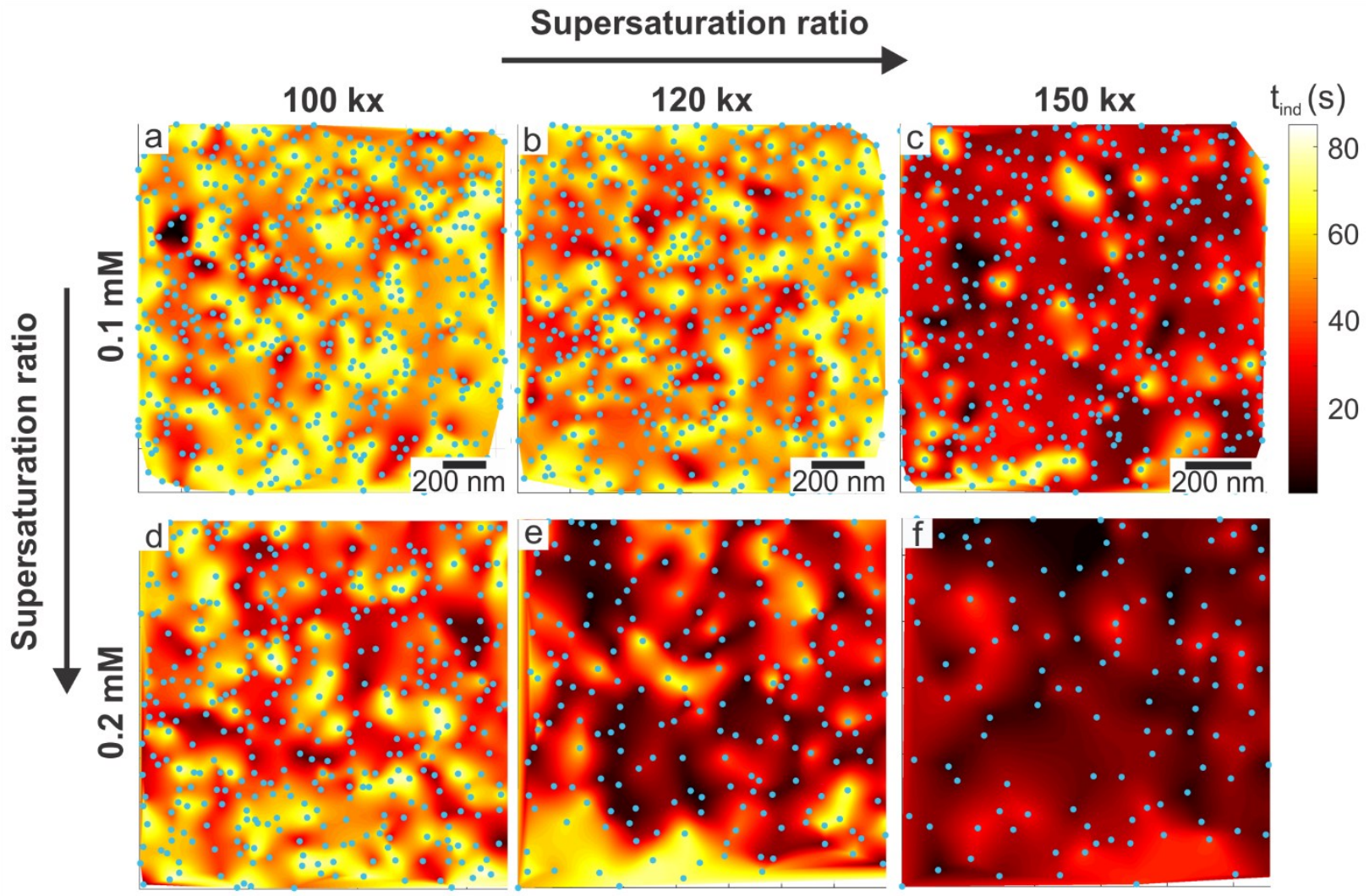

Figure S2. Static nanometric spatial maps of silver nanocrystal nucleation induction times as a function of LC-STEM magnification and precursor concentration with beam current of $21 \mathrm{pA}$. Top row: $0.1 \mathrm{mM} \mathrm{AgNO}_{3}$ at magnifications of a) $100 \mathrm{kx}(\dot{d}=0.9 \mathrm{MGy} / \mathrm{s})$, b) $120 \mathrm{kx}(\dot{d}=1.3 \mathrm{MGy} / \mathrm{s})$, and c) $150 \mathrm{kx}(\dot{d}=2.0 \mathrm{MGy} / \mathrm{s})$. Bottom row: $0.2 \mathrm{mM} \mathrm{AgNO}_{3}$ at magnifications of d) $\left.100 \mathrm{kx}, \mathrm{e}\right)$ $120 \mathrm{kx}$, and f) $150 \mathrm{kx}$. The blue dots are centroids of all silver nanocrystals formed during each LC-STEM experiment, while the color map corresponds to the interpolated local nucleation induction time. Yellow and white areas correspond to large nucleation induction times (low nucleation rate) while red and black are small nucleation induction times (high nucleation rate). 


\section{Silanization treatment and EDC-NHS coupling}

In our silanization treatment experiments, APTES was covalently bonded to surface silanol groups, leaving a pendant amine functional group on the surface for coupling with carboxylated gold nanoparticles. EDC and sulfo-NHS were mixed with the carboxylated gold particle solution. EDC forms an unstable intermediate with carboxylated gold particles that reacts with sulfo NHS to form a stable amine reactive species. Mercaptoethanol was added after EDC to quench the excess EDC. The gold nanoparticles with amine reactive surface groups were then incubated with a silanized silicon nitride surface for an hour and covalently bond to the all the amine groups to mark the positions of all functional groups. See the scheme in Figure S3 for a summary of all steps involved in the process.

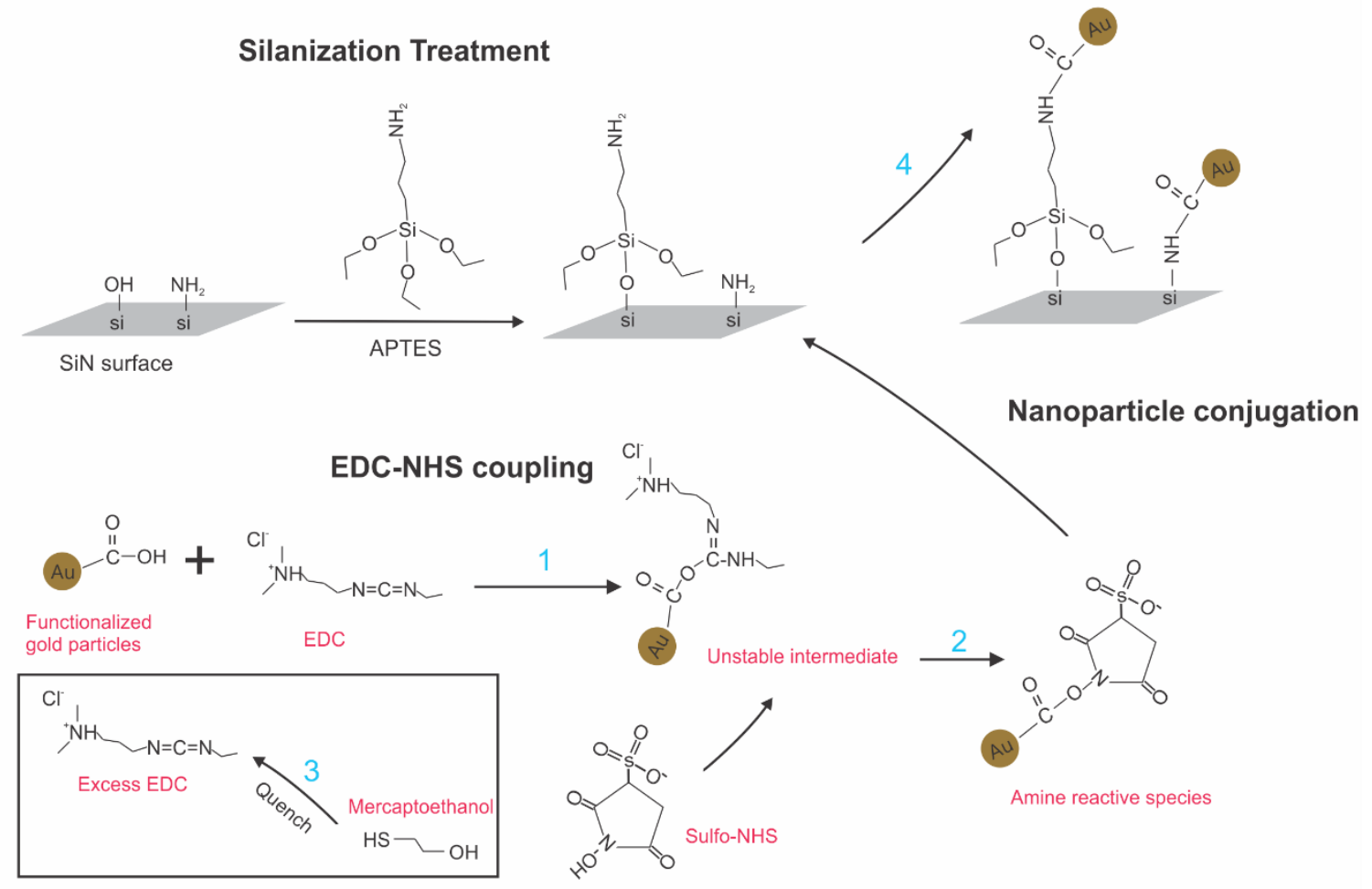

Figure S3. Surface chemistry of silanization and nanoparticle labeling for determining functional group positions.

For each labeling experiment, a silicon nitride coated LC-STEM chip was plasma cleaned for 3 minutes in an air plasma (Harrick Plasma, PDC-32G) and submerged in an aqueous 3 vol\% APTES solution at $75^{\circ} \mathrm{C}$ for an hour and then rinsed with DI water. ${ }^{7}$ Carboxylated $10 \mathrm{~nm}$ diameter gold nanoparticles $(0.05 \mathrm{mg} / \mathrm{ml}$ PEG - carboxyl NPs, nanoComposix, San Diego, CA, USA) were conjugated to the surface amine groups using EDC-NHS coupling as described above to mark the 
positions of silanol and silamine functional groups. Chips are thoroughly washed in DI water after labeling to remove unbound particles. Native silamine groups were labeled by binding nanoparticles to a native silicon nitride surface with no silanizaiton. TEM images of gold nanoparticle labels were taken with a JEOL JEM-2100 LaB6 TEM.

\section{Nanoparticle surface labeling control experiments}

Non-specific binding. To test whether non-specific binding of nanoparticles caused the domains on the silicon nitride surface observed in Figure 4c,d, we performed control experiments. Silicon nitride membranes were submerged in DI water without APTES at $75{ }^{\circ} \mathrm{C}$ for an hour and then submerged in carboxylated $10 \mathrm{~nm}$ diameter gold nanoparticles solution $(0.05 \mathrm{mg} / \mathrm{ml}$ PEG carboxyl NPs, nanoComposix, San Diego, CA, USA) for 2 hours. The chips were then thoroughly rinsed in DI water. TEM images showed random binding of nanoparticles to the surface and absence of defined surface domains (Figure S4), indicating non-specific binding does not cause formation of surface domains.

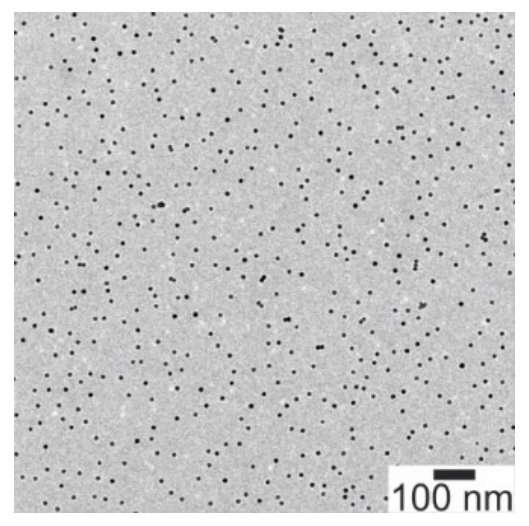

Figure S4. TEM image of non-specific binding of carboxylated gold nanoparticles to a native silicon nitride membrane surface

Silanol and silamine functional group coverage on native silicon nitride. Silicon nitride membranes not treated with plasma were silanized and labeled with gold nanoparticles to mark the positions of all natural silamine and silanol functional groups (Figure S5a). Silicon nitride membranes not treated with plasma or silanization were labeled with gold nanoparticles to mark the positions of only native silamine groups (Figure S5b). TEM images of the labeled surface showed the silamine coverage on the silicon nitride surface was at least 5 times lower compared with the total functional group coverage. 


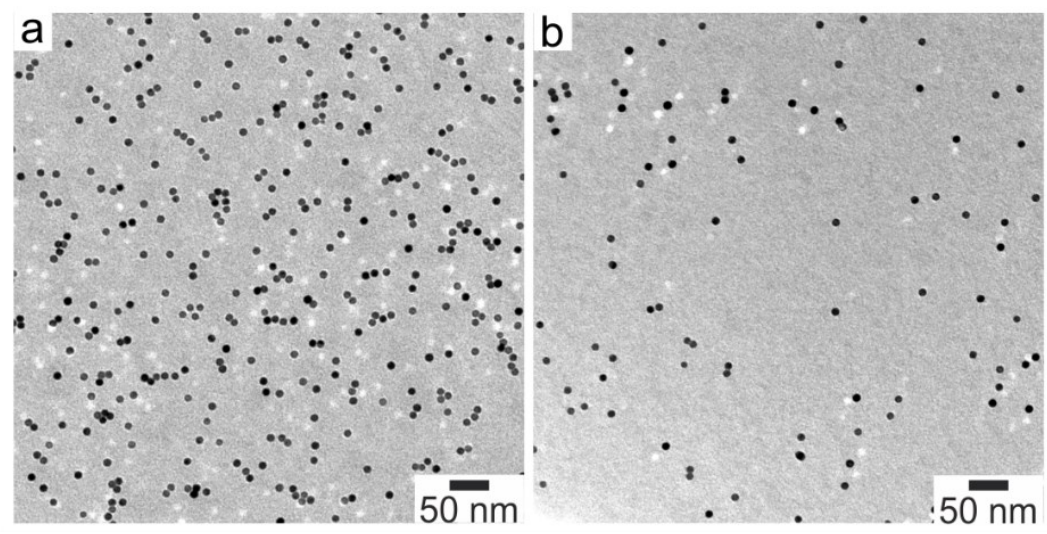

Figure S5. TEM images of nanoparticle labeled functional groups on native silicon nitride without plasma treatment. a) Silanol and natural silamine functional groups on silicon nitride. b) Natural silamine functional groups on silicon nitride.

$\mathrm{SiO}_{2}$ membrane functional group labeling. Nanoparticle labeling of silanol groups on silicon dioxide was done on a $40 \mathrm{~nm}$ free standing $\mathrm{SiO}_{2}$ membrane using the same substrate preparation conditions and labeling method at the silicon nitride membranes. (Figure S6 a,b).

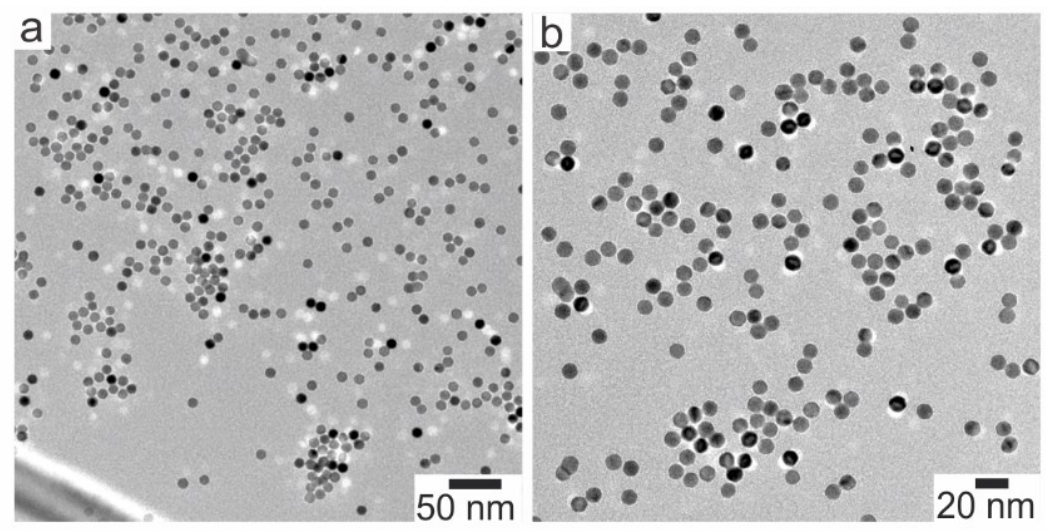

Figure S6. TEM images of gold nanoparticle labeled silanol functional groups on silicon dioxide surface.

\section{Calculations of functional groups on silicon nitride membrane}

The silicon nitride membranes possess silamine $\left(\mathrm{Si}-\mathrm{NH}_{2}\right)$ and silanol $(\mathrm{Si}-\mathrm{OH})$ functional groups that are protonated and deprotonated as $\mathrm{pH}$ changes. Functional groups are in equilibrium with protons in water through the following reactions: ${ }^{8}$ 


$$
\begin{gathered}
\mathrm{Si}-\mathrm{OH}_{2}^{+}+\mathrm{H}_{2} \mathrm{O} \leftrightarrow \mathrm{Si}-\mathrm{OH}+\mathrm{H}_{3} \mathrm{O}^{+} \\
\mathrm{Si}-\mathrm{OH}+\mathrm{H}_{2} \mathrm{O} \leftrightarrow \mathrm{Si}-\mathrm{O}^{-}+\mathrm{H}_{3} \mathrm{O}^{+} \\
\mathrm{Si}-\mathrm{NH}_{3}^{+}+\mathrm{H}_{2} \mathrm{O} \leftrightarrow \mathrm{Si}-\mathrm{NH}_{2}+\mathrm{H}_{3} \mathrm{O}^{+}
\end{gathered}
$$

Here we derive the fraction of each species as a function of $\mathrm{pH}$, with known dissociation constants $p K a_{S 2}=0.1, p K a_{S 3}=4.75$, and $p K a_{S 4}=11$. Let $N_{\text {total }}$ be the total number per surface area of all functional groups, $x_{\text {silanol }}$ the fraction of silanol functional groups in all states and $x_{\text {silamine }}$ the fraction of silamine functional groups in all states. The number of each functional group species per surface area can be calculated as

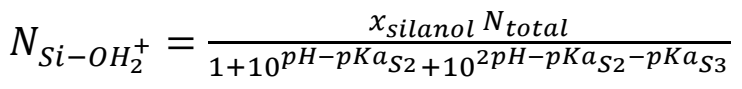

$$
\begin{aligned}
& N_{S i-O^{-}}=\frac{x_{\text {silanol }} N_{\text {total }}}{1+10^{p K a_{S 3}-p H}+10^{p K a_{S 2}+p K a_{S 3}-2 p H}} \\
& N_{S i-O H}=\frac{x_{\text {silanol }} N_{\text {total }}}{1+10^{p K a_{S 2}-p H}+10^{p H-p K a_{S 3}}} \\
& N_{S i-N H_{3}^{+}}=\frac{x_{\text {silamine }} N_{\text {total }}}{1+10^{p H-p K a_{S 4}}} \\
& N_{S i-N H_{2}}=\frac{x_{\text {silamine }} N_{\text {total }}}{1+10^{p K a_{S 4}-p H}}
\end{aligned}
$$

Here, according to our previous result, $x_{\text {silanol }} \gg x_{\text {silamine }}$, the fraction of each species can be calculated with the result shown in Figure S7.

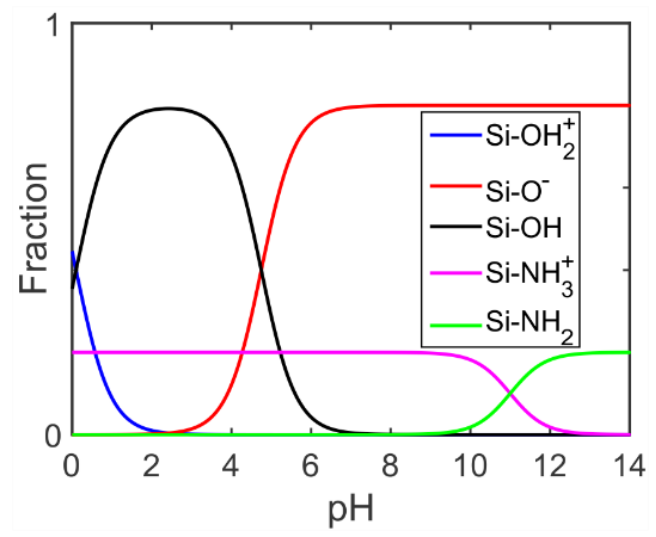

Figure S7. The fraction of different protonation functional groups on silicon nitride membrane as a function of $\mathrm{pH}$.

\section{Atomic force microscopy experimental methods and control experiments.}

The AFM and CFM experiments were performed on an Asylum Cypher ES instrument. The probe was a Nanoworld Arrow UHFAuD probe with a resonant frequency of $1.1 \mathrm{MHz}$. The AFM was operated at a large set point and small drive amplitude using plasma treated hydrophilic 
silicon nitride cantilever and silicon nitride membranes to achieve CFM imaging. ${ }^{9} 0.1 \mathrm{M}$ tertbutanol solution was degassed by argon bubble for one hour and then drop-cast on the silicon nitride membrane. All AFM measurements were carried out in tapping mode. The AFM data shown in the main text was acquired using a plasma treated silicon nitride membrane and AFM cantilever. As a control experiment, we performed AFM without plasma treating the silicon nitride membrane or the AFM cantilever (Figure S8). In comparison with Figure 4a,b in main text, we can see that AFM images acquired without plasma treatment showed essentially no heterogeneity in the CFM phase image. This provides further evidence that plasma treatment causes formation of surface domains of functional groups.

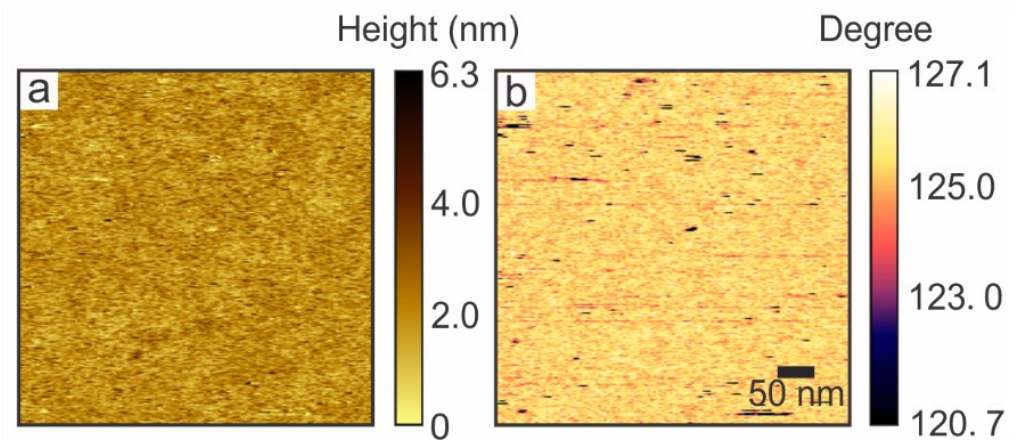

Figure S8. Tapping mode liquid phase AFM height image (a) and CFM phase image (b) of nonplasma treated SiN membrane.

\section{Classical nucleation theory calculations.}

To determine the approximate homogeneous nucleation energy barriers to use in our model calculations, we employed a model developed by Privman et al. for burst nucleation of gold nanoparticles during colloidal synthesis. ${ }^{10}$ Briefly, expressions for the critical number of atoms in a nucleus $\left(n_{c r i t}\right)$ and the associated critical free energy barrier to homogeneous nucleation $\left(\Delta G_{\text {hom }}\right)$ are

$$
\begin{aligned}
& n_{\text {crit }}(S)=\left(\frac{8 \pi a^{2} \sigma_{A g-W}}{3 k_{B} T \ln (S)}\right)^{3} \\
& \Delta G_{\text {hom }}(S)=\frac{256 \pi^{3} a^{6} \sigma_{A g-W}^{3}}{27\left(k_{B} \operatorname{Tln}(S)\right)^{2}} .
\end{aligned}
$$

Here $a$ is the atomic radius of silver $(a=172 \mathrm{pm}), \sigma_{A g-W}$ is interfacial energy between water and silver nucleus, $S$ is the supersaturation ratio $S=\frac{c}{c_{0}}, c$ is the concentration of silver atoms 
in the liquid phase, and $c_{0}$ is the equilibrium solubility of silver. The equilibrium solubility of silver is taken to be $c_{0}=2 * 10^{-12} M^{11}$ and the interfacial energy was estimated to be $\sigma_{A g-W}=$ $0.42 \frac{\mathrm{N}}{\mathrm{m}}$ based on an empirical correlation derived by Bennema and Sohnel. ${ }^{12}$ The concentration of silver atoms cannot be determined exactly, so we used a range of values up to a starting $\mathrm{Ag}^{+}$ concentration of $100 \mu M, c=10-100 \mu \mathrm{M}$. A range of silver atom concentrations is given because this value will vary with beam induced growth conditions, magnification and dose rate. With this information, we estimate the range of homogeneous nucleation free energy barriers and assess whether the numbers make physical sense by examining $n_{\text {crit }}$ and $\Delta G_{\text {hom }}$. Figure $\mathbf{S 9}$ shows plots of these values for the range of silver concentrations given. Despite the often-poor agreement of CNT with experimental systems, the values returned are reasonable. The homogeneous free energy barrier ranges from $28-36 k_{b} T$ and the critical nucleus size ranges from about $3-5$ atoms. Assuming the nuclei are spherical and have the same physical properties as bulk silver, we estimate the critical nucleus radius to range between $0.23-0.27 \mathrm{~nm}$.
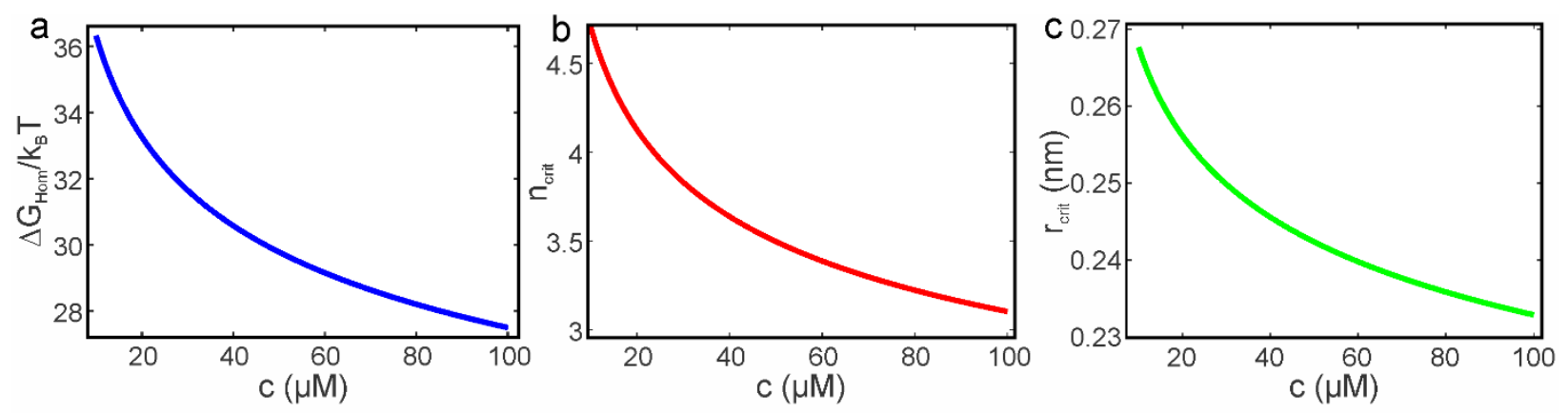

Figure S9. Classical nucleation theory calculation for silver nucleation. (a) The free energy barrier for homogeneous nucleation as a function of silver concentration. (b) The number of atoms in a critical nucleus as a function of silver concentration. (c) The estimated critical nucleus radius as a function of silver concertation.

To determine the heterogeneous nucleation free energy barrier, nucleation barrier reduction factors were randomly chosen from two normal probability distribution functions, $p\left(\phi_{i, 1}\right)$ and $p\left(\phi_{i, 2}\right)$. The mean and standard deviation for $p\left(\phi_{i, 1}\right)$ were $\mu=0.4$ and $\sigma=0.01$ and the mean and standard deviation of $p\left(\phi_{i, 2}\right)$ was $\mu=0.5$ and $\sigma=0.01$. These values were chosen to give a minimum free energy barrier height of $10 k_{B} T$ and to give comparative values of nucleation rate 
that qualitatively matched trends shown in the nucleation maps in Figure 3 in the main text. 5000 values of $\phi$ were randomly selected from each distribution and used to determine the nucleation rates shown in Figures $\mathbf{3 b}$ and $\mathbf{3 c}$ in the main text.

\section{Supplementary movies}

Movie S1: LC-STEM video data set used to generate Figure 2a and Figure 3a. Magnification is $80 \mathrm{kx}$, beam current is $31 \mathrm{pA}$, and dose rate is $6.971 \times 10^{5} \mathrm{~Gy} / \mathrm{s}$. The pixel size is $0.176 \mathrm{pixel} / \mathrm{nm}$ and the video is displayed at 5 times real time.

Movie S2: LC-STEM video data set used to generate Figure 2b and Figure 3b. Magnification is $100 \mathrm{kx}$, beam current is $31 \mathrm{pA}$, and dose rate is $1.329 \times 10^{6} \mathrm{~Gy} / \mathrm{s}$. The pixel size is $0.243 \mathrm{pixel} / \mathrm{nm}$ and the video is displayed at 5 times real time.

Movie S3: LC-STEM video data set used to generate Figure 2c and Figure 3c. Magnification is $150 \mathrm{kx}$, beam current is $31 \mathrm{pA}$, and dose rate is $2.982 \times 10^{6} \mathrm{~Gy} / \mathrm{s}$. The pixel size is $0.364 \mathrm{pixel} / \mathrm{nm}$ and the video is displayed at 5 times real time.

Movie S4: Full data set of videos frames shown in Figure 2a. Frame rate is 5 fps. The units are in Nuclei/nm ${ }^{2} s$.

Movie S5: Full data set of videos frames shown in Figure 2b. Frame rate is $5 \mathrm{fps}$. The units are in Nuclei/ $\mathrm{nm}^{2} s$.

Movie S6: Full data set of videos frames shown in Figure 2c. Frame rate is 5 fps. The units are in Nuclei/ $\mathrm{nm}^{2} s$.

\section{Reference}

1. Wang, M.; Park, C.; Woehl, T. J., Quantifying the Nucleation and Growth Kinetics of Electron Beam Nanochemistry with Liquid Cell Scanning Transmission Electron Microscopy. Chemistry of Materials 2018, 30 (21), 7727-7736.

2. Alloyeau, D.; Dachraoui, W.; Javed, Y.; Belkahla, H.; Wang, G.; Lecoq, H.; Ammar, S.; Ersen, O.; Wisnet, A.; Gazeau, F.; Ricolleau, C., Unravelling Kinetic and Thermodynamic 
Effects on the Growth of Gold Nanoplates by Liquid Transmission Electron Microscopy. Nano Letters 2015, 15 (4), 2574-2581.

3. Vo, G. D.; Park, C., Robust regression for image binarization under heavy noise and nonuniform background. Pattern Recognition 2018, 81, 224-239.

4. Park, C.; Woehl, T. J.; Evans, J. E.; Browning, N. D., Minimum Cost Multi-Way Data Association for Optimizing Multitarget Tracking of Interacting Objects. IEEE Transactions on Pattern Analysis and Machine Intelligence 2015, 37 (3), 611-624.

5. Møller, J.; Syversveen, A. R.; Waagepetersen, R. P., Log Gaussian Cox Processes. Scandinavian Journal of Statistics 1998, 25 (3), 451-482.

6. Sibson, R., A brief description of natural neighbour interpolation. Interpreting multivariate data / edited by Vic Barnett 1981.

7. Robertson, A. W.; Zhu, G.; Mehdi, B. L.; Jacobs, R. M. J.; De Yoreo, J.; Browning, N. D., Nanoparticle Immobilization for Controllable Experiments in Liquid-Cell Transmission Electron Microscopy. ACS Applied Materials \& Interfaces 2018, 10 (26), 22801-22808.

8. Cloarec, J.-P.; Chevalier, C.; Genest, J.; Beauvais, J.; Chamas, H.; Chevolot, Y.; Baron, T.; Souifi, A., pH driven addressing of silicon nanowires onto Si3N4/SiO2micro-patterned surfaces. Nanotechnology 2016, 27 (29), 295602.

9. $\quad$ Noy, A.; Sanders, C. H.; Vezenov, D. V.; Wong, S. S.; Lieber, C. M., ChemicallySensitive Imaging in Tapping Mode by Chemical Force Microscopy: Relationship between Phase Lag and Adhesion. Langmuir 1998, 14 (7), 1508-1511.

10. Robb, D. T.; Privman, V., Model of Nanocrystal Formation in Solution by Burst Nucleation and Diffusional Growth. Langmuir 2008, 24 (1), 26-35.

11. Linke, W. F.; Seidell, A., Solubilities, inorganic and metal organic compounds; a compilation of solubility data from the periodical literature. 4th ed. ed.; Van Nostrand: Princeton, N.J., 1958.

12. Bennema, P.; Söhnel, O., Interfacial surface tension for crystallization and precipitation from aqueous solutions. Journal of Crystal Growth 1990, 102 (3), 547-556. 
